\title{
IMPROVED Ni I $\log (g f)$ VALUES AND ABUNDANCE DETERMINATIONS IN THE PHOTOSPHERES OF THE SUN AND METAL-POOR STAR HD 84937
}

\author{
M. P. WOOD ${ }^{1}$, J. E. LAWLER ${ }^{1}$, C. SNEdEN ${ }^{2,3}$, AND J. J. COWAN ${ }^{4}$ \\ ${ }^{1}$ Department of Physics, University of Wisconsin, Madison, WI 53706, USA; mpwood@wisc.edu, jelawler@wisc.edu \\ 2 Department of Astronomy and McDonald Observatory, University of Texas, Austin, TX 78712, USA; chris@ verdi.as.utexas.edu \\ ${ }^{3}$ Department of Astronomy and Space Sciences, Ege University, 35100 Bornova, İzmir, Turkey \\ ${ }^{4}$ Homer L. Dodge Department of Physics and Astronomy, University of Oklahoma, Norman, OK 73019, USA; cowan@ nhn.ou.edu \\ Received 2013 November 20; accepted 2014 February 14; published 2014 March 20
}

\begin{abstract}
Atomic transition probability measurements for $371 \mathrm{Ni}$ I lines in the UV through near-IR are reported. Branching fractions from data recorded using a Fourier transform spectrometer and a new echelle spectrograph are combined with published radiative lifetimes to determine these transition probabilities. Generally good agreement is found in comparisons to previously reported Ni I transition probability measurements. Use of the new echelle spectrograph, independent radiometric calibration methods, and independent data analysis routines enable a reduction of systematic errors and overall improvement in transition probability uncertainty over previous measurements. The new Ni I data are applied to high-resolution visible and UV spectra of the Sun and metal-poor star HD 84937 to derive new, more accurate $\mathrm{Ni}$ abundances. Lines covering a wide range of wavelength and excitation potential are used to search for non-LTE effects.
\end{abstract}

Key words: atomic data - methods: laboratory: atomic - stars: abundances - stars: individual (HD 84937) - Sun: abundances

Online-only material: color figures, machine-readable tables

\section{INTRODUCTION}

Accurate atomic transition probabilities (e.g., NIST Atomic Spectra Database ${ }^{5}$ and Vienna Atomic Line Database ${ }^{6}$ ) are a critical component in the determination of stellar abundances. These abundances and their trends as a function of metallicity provide valuable information regarding the nucleosynthetic history of chemical elements in the Galaxy ${ }^{7}$. Of particular importance are old, metal-poor stars, whose abundances provide a record of the nucleosynthetic processes in the earliest generations of stars. Studies of metal-poor stars have found unexpected trends with metallicity for relative iron $(\mathrm{Fe})$-group abundances (McWilliam et al. 1995a, 1995b; McWilliam 1997; Westin et al. 2000; Cowan et al. 2002; Sneden et al. 2003; Cayrel et al. 2004; Barklem et al. 2005; Lai et al. 2008; Bonifacio et al. 2009; Roederer 2009; Suda et al. 2011; Yong et al. 2013). The [X/Fe] trends cover up to \pm 1 dex over the metallicity range from so$\operatorname{lar}([\mathrm{Fe} / \mathrm{H}] \equiv 0)$ to -4 (e.g., Figure 12 of McWilliam 1997). These trends have not been reconciled with current models of supernova (SN) yields in the early Galaxy.

While it may be that models of nucleosynthesis in the early Galaxy need to be revisited, or that standard abundance derivation techniques are inadequate for low metallicity stars, the possibility remains that laboratory atomic data are contributing to the observed trends. The use of modern techniques, including branching fraction measurements from a Fourier transform spectrometer (FTS) normalized with laser-induced fluorescence (LIF) lifetime measurements, have largely eliminated 1 dex errors in experimental atomic transition probabilities. In order to

\footnotetext{
5 Available at http://physics.nist.gov/PhysRefData/ASD/lines_form.html and http://physics.nist.gov/cgi-bin/ASBib1/Fvalbib/search_form.cgi.

6 Available at http://www.astro.uu.se/ vald/php/vald.php.

7 We adopt standard spectroscopic notations. For elements $\mathrm{X}$ and $\mathrm{Y}$, the relative abundances are written $[\mathrm{X} / \mathrm{Y}]=\log _{10}\left(N_{\mathrm{X}} / N_{\mathrm{Y}}\right)_{\text {star }}-\log _{10}\left(N_{\mathrm{X}} / N_{\mathrm{Y}}\right)_{\odot}$. For element $\mathrm{X}$, the "absolute" abundance is written $\log \varepsilon(\mathrm{X})=\log _{10}\left(N_{\mathrm{X}} / N_{\mathrm{H}}\right)+$ 12. Metallicity will be considered equivalent to the $[\mathrm{Fe} / \mathrm{H}]$ value.
}

obtain the most accurate abundances it is best to use lines that are weak enough in the photosphere of the star of interest to avoid saturation. In studies covering a wide range of metallicity ( $>2$ dex), this requires using many lines covering a range of excitation potential (E.P.) and $\log (g f)$ values which can introduce the possibility that inaccurate atomic transition probabilities are affecting the measured abundance. In higher metallicity stars, one uses weaker lines with high E.P. values in the first spectra (neutral atoms are a minor ionization stage in stars of interest). As metallicity decreases, one must switch to stronger lines with lower E.P. values and possibly to second spectra lines (singly ionized atoms are the dominant ionization stage), assuming suitable second spectra lines exist in the wavelength region being analyzed. Unfortunately, due to the energy level structure of Ni II, the majority of lines from this species occur in the vacuum-UV and one must rely primarily on $\mathrm{Ni}$ I lines for abundance determinations. The strength of these high and low E.P. lines can vary by orders of magnitude, making it difficult to accurately measure both with small uncertainties. Conversely, the observed abundance trends may be caused by failure of the one-dimensional/local thermodynamic equilibrium (1D/LTE) approximations of photospheric models, traditionally used for abundance determinations, in metal-poor stars of interest (e.g., Asplund 2005). Metal-poor giant stars are favored in these studies to provide a large photon flux for high signalto-noise $(\mathrm{S} / \mathrm{N})$, high-resolution spectra. The low-density atmospheres of giant stars, combined with the reduced electron pressure from the lower metal content, results in lower collision rates which may lead to departures from LTE. The two explanations detailed above for the unexpected trends can be investigated by improving atomic transition probabilities for $\mathrm{Fe}$-group elements. If the trends are the result of 3D/non-LTE effects, one approach is to map anomalous abundances measurements for various lines covering a range of E.P. and wavelength in a wide range of stellar types. While this is a time-consuming approach, the alternative of accurately incorporating these effects in photospheric 
models is also quite difficult. The lack of key atomic data, including cross sections and rate constants for inelastic collisions of $\mathrm{H}$ and $\mathrm{He}$ with metal atoms and ions, is a major challenge for non-LTE modeling (e.g., Asplund 2005). If the observed trends persist, even after targeted searches for non-LTE effects using improved laboratory data, it would be a strong indication that models of nucleosynthetic yields in the early Galaxy are inaccurate or incomplete and need to be revisited.

An effort is underway to reduce transition probability uncertainties of selected neutral and singly ionized Fe-group lines. The work on Mn I and Mn II (Den Hartog et al. 2011) focuses on multiplets that either cover small wavelength ranges or are Russell Saunders (LS) multiplets, and occasionally both. Multiplets covering small wavelength ranges are less affected by systematic uncertainty in the radiometric calibration, while transition strengths for LS multiplets can be checked against theoretical calculations. Given these advantages, it is possible to reduce the $\log (g f)$ uncertainties to 0.02 dex with $2 \sigma$ confidence. These small uncertainties are difficult to achieve and are only practical under favorable conditions. The recent work on Ti I (Lawler et al. 2013) and Ti II (Wood et al. 2013) instead takes a broader approach by attempting to measure every possible line connecting to upper levels with previously measured lifetimes. This approach results in a much larger set of lines with transition probability measurements, though often with higher uncertainties $(0.02$ to $\sim 0.10$ dex $)$. However, the small $(\sim 0.02$ dex $)$ uncertainties achieved by Den Hartog et al. (2011) are not required for detecting non-LTE effects in metal-poor stars. Non-LTE effects of $0.5-1$ dex are found in selected metal-poor stars but are confined to $\mathrm{Mn}$ I resonance lines connecting to the ground level (J. S. Sobeck et al. 2014, in preparation).

This work on Ni I follows the same broad approach used for Ti I and Ti II by attempting transition probability measurements for every possible line from 57 odd-parity and 9 even-parity upper levels with lifetime measurements by Bergeson \& Lawler (1993). The result is a set of $371 \log (g f)$ values covering a wide range of E.P. and wavelength, with uncertainties ranging from 0.02 dex for dominant branches to $\sim 0.12$ dex for weak branches widely separated in wavelength from the dominant branch(es). The uncertainties on dominant branches are primarily from the LIF lifetime measurements while the uncertainties on weak branches, often the most important for abundance determinations, result primarily from the branching fraction measurements. Systematic effects are the dominant source of uncertainty in branching fraction measurements, and the use of both a FTS and echelle spectrograph in our study serves to better quantify and control the systematic uncertainties.

In Sections 2 and 3 we describe our laboratory data sets from the FTS and echelle spectrograph, in Section 4 we discuss the derivation of $\mathrm{Ni}$ I branching fractions from these data, and in Section 5 we present the new transition probabilities with comparisons to previous laboratory results. Finally, in Sections 6 and 7 we apply the new Ni I data to determine the photospheric Ni abundances of the Sun and metal-poor star HD 84937, using many lines covering a range of E.P., wavelength, and $\log (g f)$ values to search for non-LTE effects.

\section{FOURIER TRANSFORM SPECTROMETER DATA}

As in much of our previous branching fraction work, this Ni I branching fraction study makes use of archived FTS data from both the $1.0 \mathrm{~m}$ FTS previously at the National Solar Observatory (NSO) on Kitt Peak and the Chelsea Instruments FT500 UV FTS at Lund University in Sweden. The NSO $1.0 \mathrm{~m}$ FTS has a large etendue (like all interferometric spectrometers), a resolution limit as small as $0.01 \mathrm{~cm}^{-1}$, wavenumber accuracy to 1 part in $10^{8}$, broad spectral coverage from the near UV to -IR, and a high data collection rate (Brault 1976). Unfortunately the NSO FTS has been dismantled, and while there are plans to restore it to full operational status at a university laboratory, it is currently unavailable to guest observers. The Chelsea Instruments FT500 has a resolution limit as small as $0.025 \mathrm{~cm}^{-1}$, wavenumber accuracy to better than 1 part in $10^{7}$, and coverage through the UV down to $1700 \AA$ (Thorne et al. 1987). Table 1 lists the 37 FTS spectra used in our Ni I branching fraction study. All NSO spectra, raw interferograms, and header files are available in the NSO electronic archives. ${ }^{8}$

Multiple FTS spectra are needed to determine high-quality branching fractions. Optimum sensitivity is achieved for different spectral ranges using various beam splitter, filter, and detector combinations. Lamps with a Ne buffer gas allow for the correction of blends between $\mathrm{Ni}$ and Ar lines. In addition, spectra are needed with lamps operating at a range of current. Overlapping visible-UV and IR spectra of the lamps operating at high currents are needed for high $\mathrm{S} / \mathrm{N}$ measurements on very weak branches to all known lower levels. Conversely, one also needs visible-UV spectra of the lamps operating at low currents in which the dominant branches are optically thin. Lines of Ni I are particularly prone to optical depth problems, even at the lowest currents available in the listed FTS spectra. The unusually severe optical depth problems are likely due to some combination of: (1) the relatively simple energy level structure of this atom which yields reduced Boltzmann factor dilution of lower level populations, (2) narrow line profiles from a near absence of hyperfine structure and small isotope shifts, (3) short upper level lifetimes which yield exceptionally strong lines to ground and low metastable levels, and (4) a high sputtering rate in the hollow cathode discharge (HCD) lamps. The optical depth concerns are addressed using the echelle spectrograph described in Section 3.

A relative radiometric calibration of the FTS is essential for the measurement of accurate emission branching fractions. As in our past branching fraction studies we make use of the Ar I and Ar II line calibration technique. Sets of well-known branching ratios for ArI and Ar II lines have been established for this purpose in the $4300-35,000 \mathrm{~cm}^{-1}$ range by Adams \& Whaling (1981), Danzmann \& Kock (1982), Hashiguchi \& Hasikuni (1985), and Whaling et al. (1993). Intensities for these lines are measured and compared to the known branching ratios in order to construct a relative radiometric calibration. This technique is internal to the HCD lamps and captures the wavelengthdependent response of the detectors, spectrometer optics, lamp windows, and any reflections which contribute to the measured signal. We can extend the calibration beyond $35,000 \mathrm{~cm}^{-1}$ by comparing measured intensities of Ni I lines in the FTS spectra to the intensities of the same lines measured with the echelle spectrograph. As described in the next section, the relative radiometric calibration of the echelle spectrograph is established using a NIST-traceable deuterium $\left(\mathrm{D}_{2}\right)$ lamp, which is routinely checked against a NIST-calibrated Ar mini-arc and provides reliable UV calibration to $50,000 \mathrm{~cm}^{-1}$.

\section{ECHELLE SPECTROGRAPH DATA}

As mentioned in the previous section, a major motivation for the construction of a powerful new echelle spectrograph at

\footnotetext{
8 Available at http://nsokp.nso.edu/.
} 
Table 1

Fourier Transform Spectra of Ni Hollow Cathode Discharge (HCD) Lamps

\begin{tabular}{|c|c|c|c|c|c|c|c|c|c|c|c|}
\hline Index & Date & $\begin{array}{c}\text { Serial } \\
\text { Number }\end{array}$ & $\begin{array}{c}\text { Lamp }^{\mathrm{a}} \\
\text { Type }\end{array}$ & $\begin{array}{c}\text { Buffer } \\
\text { Gas }\end{array}$ & $\begin{array}{l}\text { Lamp } \\
\text { Current } \\
(\mathrm{mA})\end{array}$ & $\begin{array}{c}\text { Wavenumber } \\
\text { Range } \\
\left(\mathrm{cm}^{-1}\right)\end{array}$ & $\begin{array}{l}\text { Limit of } \\
\text { Resolution } \\
\left(\mathrm{cm}^{-1}\right)\end{array}$ & & $\begin{array}{c}\text { Beam } \\
\text { Splitter }\end{array}$ & Filter & Detector $^{b}$ \\
\hline 1 & 1994 Nov 4 & 658 & Commercial HCD & $\mathrm{Ar}$ & 25 & $28436-56873$ & 0.070 & 22 & $\mathrm{SiO}_{2}$ & & R166 Solar Blind PMTs \\
\hline 2 & 1994 Nov 4 & 659 & Commercial HCD & $\mathrm{Ar}$ & 20 & $28436-56873$ & 0.070 & 22 & $\mathrm{SiO}_{2}$ & & R166 Solar Blind PMTs \\
\hline 3 & 1994 Nov 4 & 660 & Commercial HCD & $\mathrm{Ar}$ & 15 & $28436-56873$ & 0.070 & 22 & $\mathrm{SiO}_{2}$ & & R166 Solar Blind PMTs \\
\hline 4 & 1994 Nov 4 & 661 & Commercial HCD & $\mathrm{Ar}$ & 10 & $28436-56873$ & 0.070 & 22 & $\mathrm{SiO}_{2}$ & & R166 Solar Blind PMTs \\
\hline 5 & 1994 Nov 4 & 662 & Commercial HCD & $\mathrm{Ar}$ & 5 & $28436-56873$ & 0.070 & 22 & $\mathrm{SiO}_{2}$ & & R166 Solar Blind PMTs \\
\hline 6 & 1994 Nov 4 & 664 & Commercial HCD & $\mathrm{Ar}$ & 25 & $22117-44234$ & 0.050 & 22 & $\mathrm{SiO}_{2}$ & BG24 & 1P28 \& R1516 PMTs \\
\hline 7 & 1994 Nov 4 & 665 & Commercial HCD & $\mathrm{Ar}$ & 20 & $22117-44234$ & 0.050 & 22 & $\mathrm{SiO}_{2}$ & BG24 & 1P28 \& R1516 PMTs \\
\hline 8 & 1994 Nov 4 & 667 & Commercial HCD & $\mathrm{Ar}$ & 15 & $22117-44234$ & 0.040 & 22 & $\mathrm{SiO}_{2}$ & BG24 & 1P28 \& R1516 PMTs \\
\hline 9 & 1994 Nov 4 & 669 & Commercial HCD & $\mathrm{Ar}$ & 10 & 22117-44234 & 0.050 & 22 & $\mathrm{SiO}_{2}$ & $\mathrm{BG} 24$ & 1P28 \& R1516 PMTs \\
\hline 10 & 1994 Nov 4 & 670 & Commercial HCD & $\mathrm{Ar}$ & 5 & $22117-44234$ & 0.050 & 22 & $\mathrm{SiO}_{2}$ & BG24 & 1P28 \& R1516 PMTs \\
\hline 11 & 1994 Nov 4 & 671 & Commercial HCD & $\mathrm{Ar}$ & 3 & $22117-44234$ & 0.050 & 22 & $\mathrm{SiO}_{2}$ & BG23 & 1P28 \& R1516 PMTs \\
\hline 12 & 1994 Nov 5 & 674 & Commercial HCD & $\mathrm{Ar}$ & 10 & $15798-31596$ & 0.050 & 22 & $\mathrm{SiO}_{2}$ & BG23 & 1P28 \& R1516 PMTs \\
\hline 13 & 1994 Nov 5 & 677 & Commercial HCD & $\mathrm{Ar}$ & 5 & $15798-31596$ & 0.035 & 22 & $\mathrm{SiO}_{2}$ & BG23 & 1P28 \& R1516 PMTs \\
\hline 14 & 1994 Nov 4 & 673 & Commercial HCD & $\mathrm{Ar}$ & 5 & $15798-31596$ & 0.050 & 22 & $\mathrm{SiO}_{2}$ & BG23 & 1P28 \& R1516 PMTs \\
\hline 15 & 1994 Nov 4 & 672 & Commercial HCD & $\mathrm{Ar}$ & 3 & $15798-31596$ & 0.050 & 22 & $\mathrm{SiO}_{2}$ & $\mathrm{BG} 23$ & 1P28 \& R1516 PMTs \\
\hline 16 & 1985 Jul 31 & 7 & Custom HCD & $\mathrm{Ne}-\mathrm{Ar}$ & 820 & $7181-46955$ & 0.064 & 7 & UV & & Mid Range Si PDs \\
\hline 17 & 1985 Jul 31 & 9 & Custom HCD & $\mathrm{Ne}-\mathrm{Ar}$ & 80 & $7181-46955$ & 0.064 & 7 & UV & & Mid Range Si PDs \\
\hline 18 & 1979 Dec 29 & 1 & Custom HCD & Ar & 800 & 4789-39199 & 0.054 & 12 & UV & CS 9-54 & Super Blue Si PDs \\
\hline 19 & 1979 Dec 28 & 5 & Custom HCD & $\mathrm{Ar}$ & 625 & $13607-37456$ & 0.045 & 5 & UV & $\mathrm{CuSO}_{4}+\mathrm{CS} 9-54$ & Super Blue Si PDs \\
\hline 20 & 1979 Dec 27 & 1 & Custom HCD & Ar & 400 & $13607-37456$ & 0.045 & 7 & UV & $\mathrm{CuSO}_{4}+\mathrm{CS} 9-54$ & Mid Range Si PDs \\
\hline 21 & 1981 Jun 15 & 2 & Custom HCD & $\mathrm{Ar}$ & 144 & $6924-36533$ & 0.043 & 8 & UV & WG 295 & Mid Range Si PDs \\
\hline 22 & 1981 Jun 20 & 1 & Custom HCD & $\mathrm{Ar}$ & 580 & 8986-30601 & 0.037 & 20 & UV & CS 4-97 + GG 375 & Mid Range Si PDs \\
\hline 23 & 1981 Jun 17 & 1 & Custom HCD & $\mathrm{Ar}$ & 550 & $14878-36386$ & 0.043 & 8 & UV & $\mathrm{CuSO}_{4}$ & Mid Range Si PDs \\
\hline 24 & 1981 Sept 22 & 1 & Custom HCD & Ar & 670 & $18529-36203$ & 0.035 & 25 & UV & $\mathrm{CuSO}_{4}$ & Super Blue Si PDs \\
\hline 25 & 1983 Feb 24 & 2 & Custom HCD & $\mathrm{Ar}$ & 2000 & $16020-36658$ & 0.047 & 7 & UV & $\mathrm{CuSO}_{4}$ & Mid Range Si PDs \\
\hline 26 & 1983 Feb 24 & 1 & Custom HCD & $\mathrm{Ar}$ & 1000 & $16020-36658$ & 0.047 & 16 & UV & $\mathrm{CuSO}_{4}$ & Mid Range Si PDs \\
\hline 27 & 1983 Feb 12 & 8 & Custom HCD & $\mathrm{Ar}$ & 80 & $15587-36081$ & 0.044 & 8 & UV & $\mathrm{CuSO}_{4}$ & Mid Range Si PDs \\
\hline 28 & 1996 Sept 4 & 8 & Commercial HCD & $\mathrm{Ar}$ & 9 & $0-34998$ & 0.053 & 4 & UV & & Super Blue Si PDs \\
\hline 29 & 1996 Sept 4 & 9 & Commercial HCD & $\mathrm{Ar}$ & 6 & 0-34998 & 0.053 & 4 & UV & & Super Blue Si PDs \\
\hline 30 & 1996 Sept 4 & 10 & Commercial HCD & $\mathrm{Ar}$ & 3 & $0-34998$ & 0.053 & 4 & UV & & Super Blue Si PDs \\
\hline 31 & 1996 Sept 7 & 20 & Commercial HCD & $\mathrm{Ar}$ & 0.8 & $0-34998$ & 0.053 & 10 & UV & & Mid Range Si PDs \\
\hline 32 & 1980 Mar 20 & 2 & Custom HCD & Ar & 640 & 3571-9937 & 0.012 & 16 & Vis & & $\mathrm{InSb}$ \\
\hline 33 & 1983 Feb 25 & 3 & Custom HCD & Ar & 2000 & $1985-7811$ & 0.011 & 4 & UV $\quad$ & C 4300 + Wedged Ge & Mid Range Si PD + InSb \\
\hline 34 & 1983 Feb 25 & 2 & Custom HCD & Ar & 1000 & $1985-7811$ & 0.011 & 4 & UV $\quad$ & C 4300 + Wedged Ge & Mid Range Si PD + InSb \\
\hline 35 & 1984 Jul 26 & 18 & Custom HCD & Ar & 1500 & $7982-45407$ & 0.054 & 8 & UV & WG 295 & Mid Range Si PD + R166 Solar Blind \\
\hline 36 & 1981 Jun 21 & 5 & Custom HCD & $\mathrm{Ne}$ & 800 & $24449-34522$ & 0.040 & 40 & UV & $\mathrm{CuSO}_{4}+\mathrm{CS} 7-51$ & Mid Range Si PDs \\
\hline 37 & 1981 Jun 20 & 2 & Custom HCD & $\mathrm{Ne}$ & 780 & 13479-30601 & 0.037 & 18 & UV & CS 4-96 & Mid Range Si PDs \\
\hline
\end{tabular}

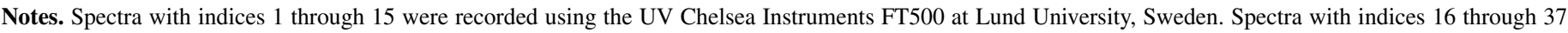
were recorded using the $1.0 \mathrm{~m}$ FTS on the McMath telescope at the National Solar Observatory, Kitt Peak, AZ.

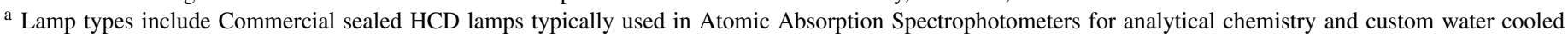
HCD lamps for high current operation.

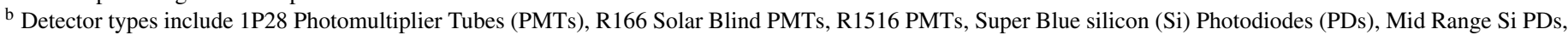
and indium antimonide ( $\mathrm{InSb}$ ) detectors for the near-infrared.

the University of Wisconsin is the closure of the NSO $1.0 \mathrm{~m}$ FTS. A further motivation is the need to reduce optical depth errors as a source of systematic uncertainty in branching fraction measurements, especially on weak lines. $\mathrm{Ni}$ is especially prone to optical depth errors and it is therefore essential to supplement the FTS data with echelle data in order to provide the most accurate atomic transition probabilities. FTS instruments suffer from multiplex noise in which quantum statistical (Poisson) noise from all spectral features, in particular the strong visible and near-IR branches, is smoothly redistributed throughout the entire spectrum. This can be a hindrance when measuring weak transitions, which are often the most important for accurate $\mathrm{Fe}$ group abundance determinations. Often, as the lamp current is reduced, the weak lines become comparable to the multiplex noise before the dominant branch(es) from the common upper level are optically thin. A dispersive spectrograph is free from multiplex noise and can provide adequate $\mathrm{S} / \mathrm{N}$ on these astrophysically important weak lines even at very low lamp current, reducing the possibility of optical depth errors.

A brief overview of the echelle spectrograph is given here. The instrument is a $3.0 \mathrm{~m}$ focal length grating spectrograph incorporating a large $(128 \times 254 \mathrm{~mm}$ ruled area), coarse (23.2 grooves $\mathrm{mm}^{-1}$ ) echelle grating blazed at 63.5. Attached to the grating spectrograph is a custom $0.5 \mathrm{~m}$ focal length order separator. The order separator is rotated into a plane orthogonal to the grating spectrograph, using a prism to separate the many overlapping high orders from the echelle grating in the transverse direction and imaging them onto a large UV-sensitive CCD (Princeton Instruments PI-SX:2048, $2048 \times 204813.5 \mu$ m pixels). The orthogonal rotation serves to 
Table 2

Echelle Spectra of Commercial Ni HCD Lamps

\begin{tabular}{|c|c|c|c|c|c|c|c|c|}
\hline Index & Date & Serial Numbers $^{\mathrm{a}}$ & Buffer Gas & $\begin{array}{l}\text { Lamp Current } \\
(\mathrm{mA})\end{array}$ & $\begin{array}{c}\text { Wavelength Range } \\
(\AA)\end{array}$ & Resolving Power ${ }^{b}$ & Coadds & $\begin{array}{c}\text { Expos. Time } \\
\text { (s) }\end{array}$ \\
\hline $38-42$ & 2012 Dec 5 & $1,3,5,7,9$ & $\mathrm{Ne}$ & 1 & $2200-3900$ & 250,000 & 44 & 120 \\
\hline $43-47$ & 2012 Dec 10 & $1,3,5,7,9$ & $\mathrm{Ne}$ & 2 & $2200-3900$ & 250,000 & 60 & 90 \\
\hline $48-52$ & 2012 Dec 4 & $11,13,15,17,19$ & $\mathrm{Ne}$ & 3 & $2200-3900$ & 250,000 & 40 & 60 \\
\hline $53-57$ & $2012 \operatorname{Dec} 4$ & $1,3,5,7,9$ & $\mathrm{Ne}$ & 5 & $2200-3900$ & 250,000 & 40 & 60 \\
\hline $58-62$ & 2012 Dec 17 & $1,3,5,7,9$ & $\mathrm{Ar}$ & 1 & $2200-3900$ & 250,000 & 6 & 900 \\
\hline $63-67$ & 2012 Dec 18 & $1,3,5,7,9$ & $\mathrm{Ar}$ & 2 & $2200-3900$ & 250,000 & 6 & 900 \\
\hline $68-72$ & 2012 Dec 19 & $1,3,5,7,9$ & $\mathrm{Ar}$ & 3 & $2200-3900$ & 250,000 & 18 & 300 \\
\hline $73-77$ & 2013 Jan 4 & $1,3,5,7,9$ & $\mathrm{Ar}$ & 5 & $2200-3900$ & 250,000 & 36 & 150 \\
\hline $78-82$ & 2013 Jan 31 & $1,3,5,7,9$ & $\mathrm{Ne}$ & 1 & $2200-3900$ & 125,000 & 80 & 90 \\
\hline $83-87$ & 2013 Feb 2 & $1,3,5,7,9$ & $\mathrm{Ne}$ & 1 & $2200-3900$ & 125,000 & 80 & 90 \\
\hline $88-92$ & 2013 Jan 29 & $1,3,5,7,9$ & $\mathrm{Ar}$ & 1 & $2200-3900$ & 125,000 & 6 & 1200 \\
\hline $93-97$ & 2013 Jan 30 & $1,3,5,7,9$ & $\mathrm{Ar}$ & 1 & $2200-3900$ & 125,000 & 6 & 1200 \\
\hline $98-102$ & 2013 Feb 13 & $1,3,5,7,9$ & $\mathrm{Ne}$ & 2 & $2000-2800$ & 250,000 & 12 & 600 \\
\hline $103-107$ & 2013 Feb 14 & $1,3,5,7,9$ & $\mathrm{Ne}$ & 3 & $2000-2800$ & 250,000 & 40 & 180 \\
\hline $108-112$ & 2013 Feb 15 & $1,3,5,7,9$ & $\mathrm{Ne}$ & 5 & $2000-2800$ & 250,000 & 80 & 90 \\
\hline $113-117$ & 2013 Feb 16 & $1,3,5,7,9$ & $\mathrm{Ar}$ & 5 & $2000-2800$ & 250,000 & 24 & 300 \\
\hline
\end{tabular}

Notes.

${ }^{\text {a }}$ At least three CCD frames are needed to capture a complete echelle grating order in the UV. In the above data five CCD frames are used to provide redundancy and a check for lamp drift.

${ }^{\mathrm{b}}$ Resolving power is adjusted by changing the diameter of the precision-machined entrance pinholes from $50 \mu \mathrm{m}$ (which gives $\left.R \sim 250,000\right)$ to $100 \mu \mathrm{m}$ $(R \sim 125,000)$.

compensate for the inherent astigmatism of the $3.0 \mathrm{~m}$ grating spectrograph, allowing the entrance slits to be replaced with precision machined pinholes. The echelle spectrograph has a resolving power of 250,000 with a $50 \mu \mathrm{m}$ entrance pinhole, broad wavelength coverage with a few CCD frames, and superb UV sensitivity. While the instrument has the advantage of being free from multiplex noise, it does suffer from reduced resolution and wavenumber precision compared to a FTS. The complete design and performance of the echelle spectrograph, including a detailed aberration analysis, is provided by Wood \& Lawler (2012).

In addition to the 37 FTS spectra listed in Table 1, the 80 CCD frames of spectra from commercial Ni HCD lamps listed in Table 2 are part of this Ni I branching fraction study. These spectra are radiometrically calibrated using a NIST-traceable $\mathrm{D}_{2}$ standard lamp to provide a UV continuum. This lamp is periodically checked against a NIST-calibrated Ar mini-arc to ensure an accurate UV calibration. The use of standard lamps to calibrate a FTS is often difficult due to ghosts, and instead we rely on the calibration method described in Section 2, but it is our preferred method for calibrating the echelle spectrograph. The use of a standard lamp also allows for branching fraction measurements to much shorter wavelengths than is achievable using ArI and ArII branching ratios since the lamps are calibrated at wavelengths down to $2000 \AA$ A.

\section{Ni I BRANCHING FRACTIONS}

All possible transitions wavenumbers between known energy levels from the 2013 NIST Atomic Spectra Database (Kramida et al. 2013) that satisfy both the parity change and $|\Delta J| \leqslant 1$ selection rules are computed and used during this branching fraction analysis. Transitions which violate these two selection rules are suppressed by a factor of $\sim 10^{6}$ and are typically unimportant for stellar abundance studies. These two selection rules are obeyed throughout the periodic table whereas many important Fe-group transitions violate the $\Delta S=0$ and $|\Delta L| \leqslant 1$ selection rules of LS coupling. We can make measurements for branching fractions as weak as 0.0001 , and therefore systematic errors from missing branches are negligible in this study. Ni has five naturally occurring isotopes, one of which $\left({ }^{61} \mathrm{Ni}\right.$, nuclear spin $I=3 / 2$ ) has hyperfine structure due to a non-zero nuclear spin. However, this isotope has a low solar system abundance of $1.1 \%$, and Ni I lines are rather narrow in the FTS data. As a result, component structure is neglected in this branching fraction study.

Branching fraction measurements are attempted for all 57 odd-parity and 9 even-parity upper levels with lifetime measurements by Bergeson \& Lawler (1993) and are completed for 52 odd-parity and all 9 even-parity levels. We note that branching fractions for the 9 even-parity upper levels were reported earlier by Wickliffe \& Lawler (1997) using a subset of the FTS data employed in this work. Levels for which branching fraction measurements could not be completed have a strong branch with a severe blending problem. As in our previous work, thousands of possible spectral line observations are analyzed in both FTS and echelle spectra to calculate the branching fractions. Integration limits and non-zero baselines are set "interactively" during data analysis. Non-zero baselines are necessary for the echelle spectra, which are not dark-signal corrected, and are occasionally needed for the FTS spectra when a line falls on the wing of a dominant feature. A simple numerical integration technique is used to determine un-calibrated Ni I line intensities because of unresolved isotopic and/or hyperfine structure that causes variations in the observed line widths. This same integration technique is also used on selected Ar I and Ar II lines to establish a relative radiometric calibration of the FTS spectra.

Branching fraction uncertainties depend on the $S / N$ of the data, the line strengths, and the wavelength separation of lines from a common upper level. Branching fraction uncertainty always migrates to the weakest lines because branching fractions sum to 1.0 by definition. Uncertainties on weak lines near the dominant branch(es) from the common upper level tend to be limited by $\mathrm{S} / \mathrm{N}$. For lines that are widely separated in wavelength from the dominant branch(es), systematic errors 
Table 3.

Experimental Atomic Transition Probabilities for 371 Lines of Ni I Organized by Increasing Wavelength in Air

\begin{tabular}{|c|c|c|c|c|c|c|c|c|}
\hline \multirow{2}{*}{$\begin{array}{l}\text { Wavelength in } \text { Air }^{\mathrm{a}} \\
(\AA)\end{array}$} & \multicolumn{3}{|c|}{ Upper Level } & \multicolumn{3}{|c|}{ Lower Level } & \multirow{2}{*}{$\begin{array}{l}\text { Transition Probability } \\
\qquad\left(10^{6} \mathrm{~s}^{-1}\right)\end{array}$} & \multirow[t]{2}{*}{$\log _{10}(g f)$} \\
\hline & Energy $^{\mathrm{b}}\left(\mathrm{cm}^{-1}\right)$ & Parity & $J$ & Energy $^{\mathrm{b}}\left(\mathrm{cm}^{-1}\right)$ & Parity & $J$ & & \\
\hline 2121.3903 & 47328.784 & od & 2 & 204.787 & ev & 3 & $24.6 \pm 1.3$ & -1.08 \\
\hline 2125.6261 & 47030.102 & od & 3 & 0.000 & ev & 4 & $3.54 \pm 0.24$ & -1.77 \\
\hline 2129.9541 & 47139.337 & od & 2 & 204.787 & ev & 3 & $3.53 \pm 0.24$ & -1.92 \\
\hline 2134.9235 & 47030.102 & od & 3 & 204.787 & ev & 3 & $10.1 \pm 0.7$ & -1.32 \\
\hline 2147.7838 & 47424.785 & od & 1 & 879.816 & ev & 2 & $45.4 \pm 2.7$ & -1.03 \\
\hline
\end{tabular}

Notes.

${ }^{a}$ Wavelength values computed from energy levels using the standard index of air from Peck \& Reeder (1972).

${ }^{\mathrm{b}}$ Energy levels, parities, and $J$ values are from online 2013 NIST Atomic Spectra Database by Kramida et al.

(This table is available in its entirety in a machine-readable form in the online journal. A portion is shown here for guidance regarding its form and content.)

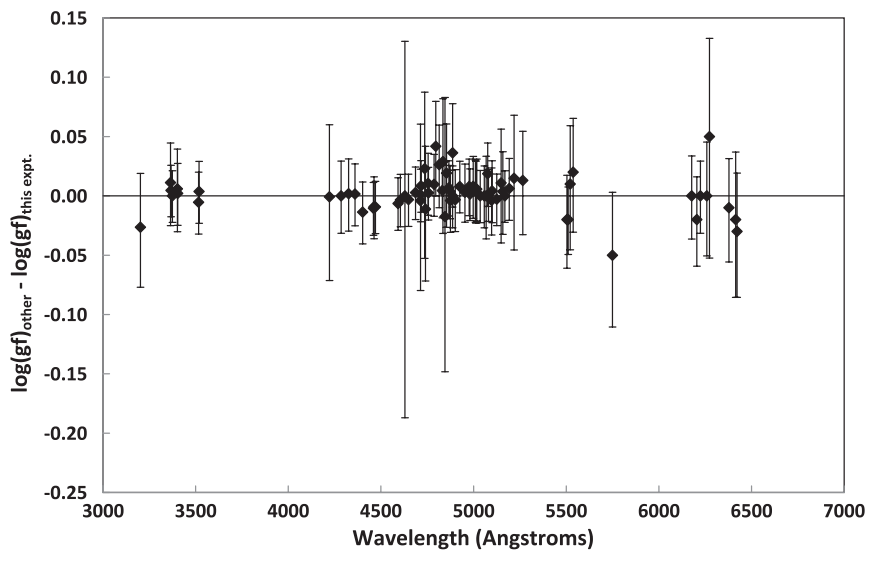

Figure 1. Comparison of $\log (g f)$ s from Wickliffe \& Lawler (1997) to results of this study as a function of wavelength for 76 lines in common. The solid horizontal line indicates perfect agreement. Wickliffe \& Lawler use a subset of the FTS data used in this work for branching fraction measurements and use the same lifetime measurements by Bergeson \& Lawler (1993) for an absolute normalization. The $\log (g f)$ differences are computed from the Einstein $A$ coefficients to reveal small differences that are obscured by truncating the $\log (g f)$ values to 0.01 dex. The error bars are from this study only.

in the radiometric calibration tend to be the dominant source of uncertainty. The systematic uncertainty in the calibration is estimated using the product of $0.001 \% \mathrm{~cm}^{-1}$ and the wavenumber difference between the line of interest and the dominant branch from the common upper level, as presented and tested by Wickliffe et al. (2000). The calibration uncertainty is combined with the standard deviation of measurements from multiple spectra to determine the total branching fraction uncertainty. The final uncertainty, especially for lines widely separated from the dominant branch(es) of the common upper level, is primarily systematic and it is therefore impractical to state whether these uncertainties represent $1 \sigma$ or $2 \sigma$ error bars. The use of data from both the FTS and echelle spectrograph, combined with independent radiometric calibration techniques, is important in assessing and controlling systematic uncertainty.

\section{Ni I TRANSITION PROBABILITIES AND COMPARISON TO EARLIER MEASUREMENTS}

Absolute transition probabilities are given for 371 lines of Ni I in Table 3. Branching fraction measurements from a combination of FTS and echelle data are normalized with published radiative lifetimes (Bergeson \& Lawler 1993). Air wavelengths in the table are computed using the standard index

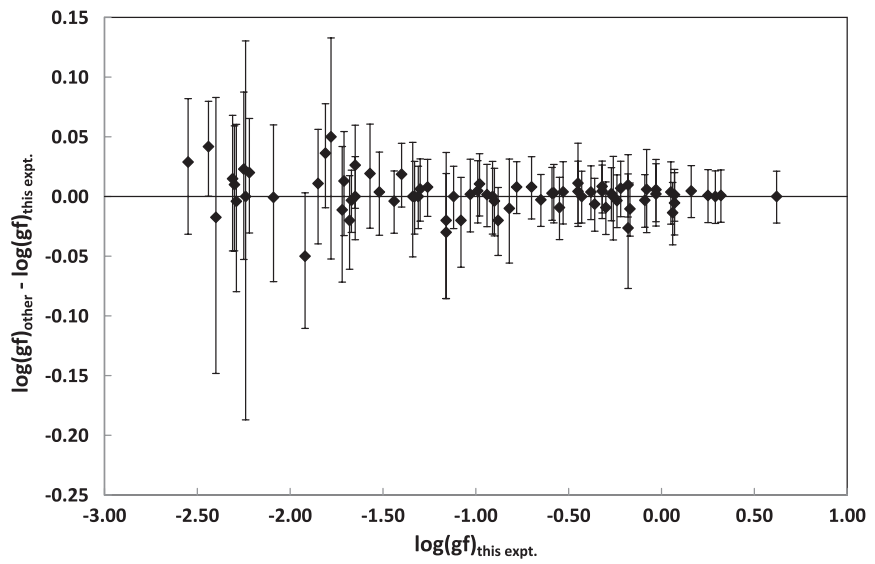

Figure 2. Comparison of $\log (g f)$ s from Wickliffe \& Lawler (1997) to results of this study as a function of $\log (g f)$ from this work. See Figure 1 for additional details.

of air (Peck \& Reeder 1972) and Ni I energy levels from the 2013 NIST Atomic Spectra Database (Kramida et al. 2013).

Often lines must be omitted if they are too weak to have reliable $\mathrm{S} / \mathrm{N}$, have uncertain classifications, or are too seriously blended to be separated. The effect of these problem lines can be seen by summing all transition probabilities for a given upper level in Table 3 and comparing the sum to the inverse upper level lifetime (Bergeson \& Lawler 1993). The sum is typically $>90 \%$ of the inverse level lifetime. While these problem lines have large fractional uncertainty in their branching fractions, this does not have a significant effect on the uncertainties of the lines kept in Table 3. The transition probability uncertainties quoted in Table 3 are found by combining branching fraction uncertainties and radiative lifetime uncertainties in quadrature.

Figure 1 compares $\log (g f)$ values from Wickliffe \& Lawler (1997) to results of this study as a function of wavelength for 76 lines in common. The horizontal line represents perfect agreement and the error bars are from this work only. Figure 2 shows the same 76 lines plotted as a function of the $\log (g f)$ measured in this study. These lines from high-lying even-parity upper levels are relatively easy to measure because they are not affected by optical depth errors and each multiplet is confined to a relatively small wavelength region. Wickliffe \& Lawler use a subset of the FTS data used in this study for branching measurements and use the same lifetime measurements by Bergeson \& Lawler (1993) for an absolute normalization, and as such, the agreement is very good. The use of an expanded set of FTS measurements in our work allows for a reduction in 


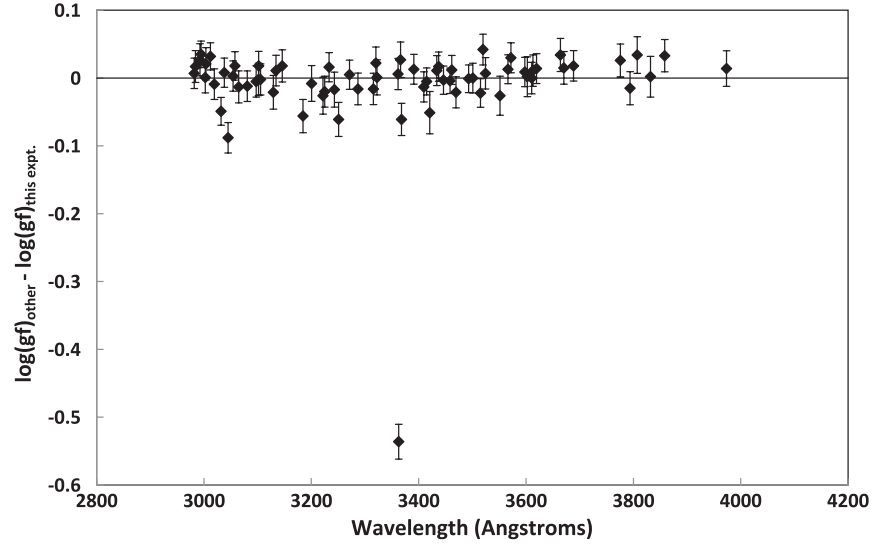

Figure 3. Comparison of $\log (g f)$ s from Blackwell et al. (1989) to results of this study as a function of wavelength for 70 lines in common. The solid horizontal line indicates perfect agreement. Blackwell et al. claim exceptional relative accuracy (about $\pm 0.7 \%$ ) for their absorption data, but claim only about $\pm 7 \%$ absolute accuracy. We have added a correction of $+0.024 \mathrm{dex}$ (about $5 \%$ ) to their results in the plot in order to clarify relative differences. The error bars are from this work only.

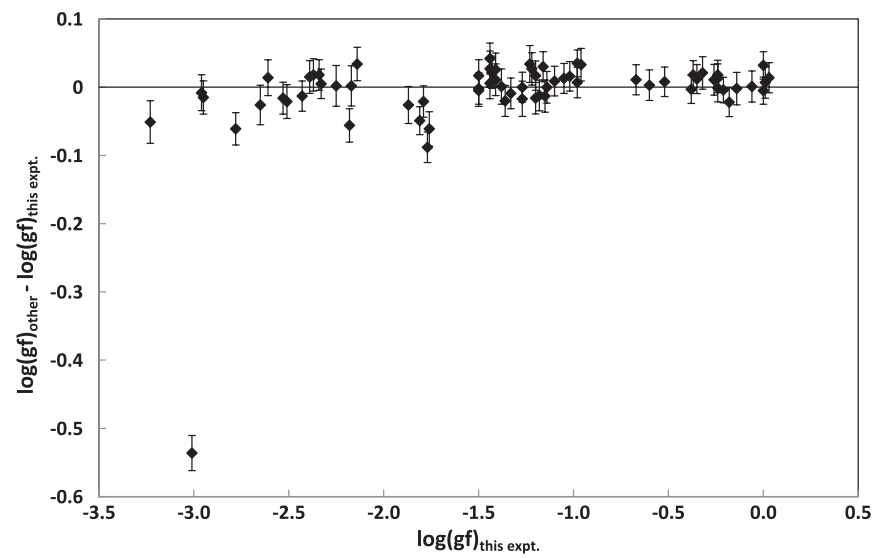

Figure 4. Comparison of $\log (g f)$ s from Blackwell et al. (1989) to results of this study as a function of $\log (g f)$ from this work. See Figure 3 for additional details.

transition probability uncertainties compared to the Wickliffe \& Lawler measurements and also facilitates the measurement of additional lines.

Figure 3 compares $\log (g f)$ values as a function of wavelength for 70 lines in common between this work and the measurements of Blackwell et al. (1989). The horizontal line indicates perfect agreement and the error bars represent uncertainties from this study only. Blackwell et al. claim exceptional relative accuracy (about $\pm 0.7 \%$ ) for their absorption data, but claim only around $\pm 7 \%$ absolute accuracy. We have added a correction of +0.024 dex (about $5 \%$ ) to their results in the figure in order to clarify the relative differences. Figure 4 shows the same 70 lines in common plotted as a function of the $\log (g f)$ measured in this study. The outlier at $3362.8 \AA$ is both well centered and has reliable $\mathrm{S} / \mathrm{N}$ in our data and we detect no problem with our measured value.

Figures 5-8 are comparisons of our measured Ni I transition probabilities to the 2013 NIST Atomic Spectra Database (Kramida et al. 2013). The database assigns an accuracy grade to each transition probability, and for the wavelength range covered in this study, there are no Ni I lines in the database with accuracy grades of B $(\leqslant 10 \%)$ or better. Figure 5 compares transition probabilities versus wavelength for 131 lines in common

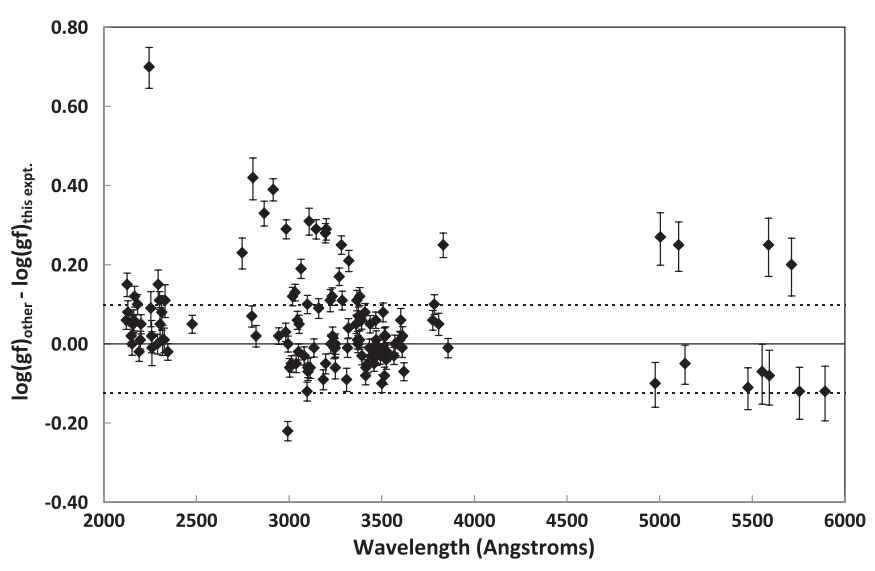

Figure 5. Comparison of $\log (g f)$ s with accuracy rank $\mathrm{C}(\leqslant 25 \%)$ and $\mathrm{C}+$ $(\leqslant 18 \%)$ from the online NIST Atomic Spectra Database by Kramida et al. (2013) (also in text by Fuhr et al. 1988) to results of this study as a function of wavelength for 131 lines in common. The solid horizontal line indicates perfect agreement and the dashed lines indicate $\pm 25 \%$ differences. The error bars are from this work only.

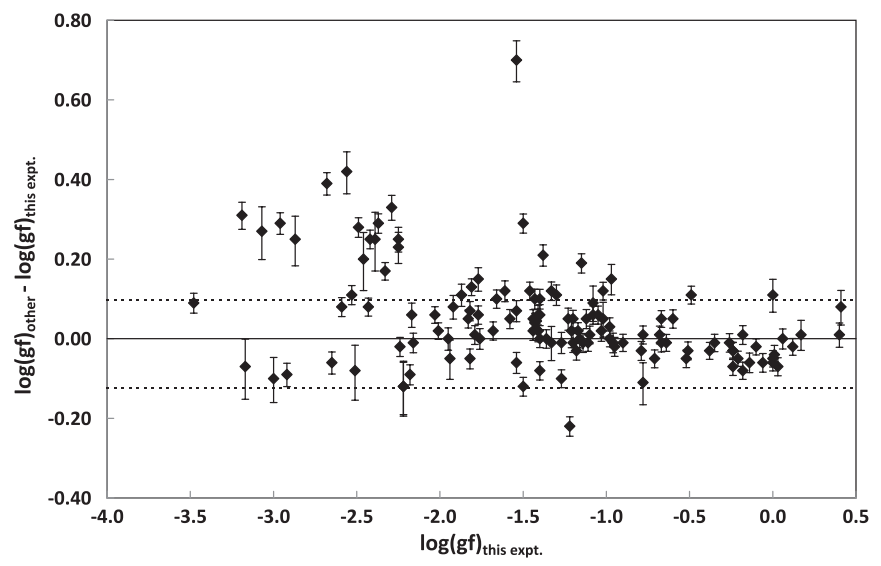

Figure 6. Comparison of $\log (g f)$ s for the same 131 lines shown in Figure 5, this time plotted as a function of $\log (g f)$ from this study. See Figure 5 for additional details.

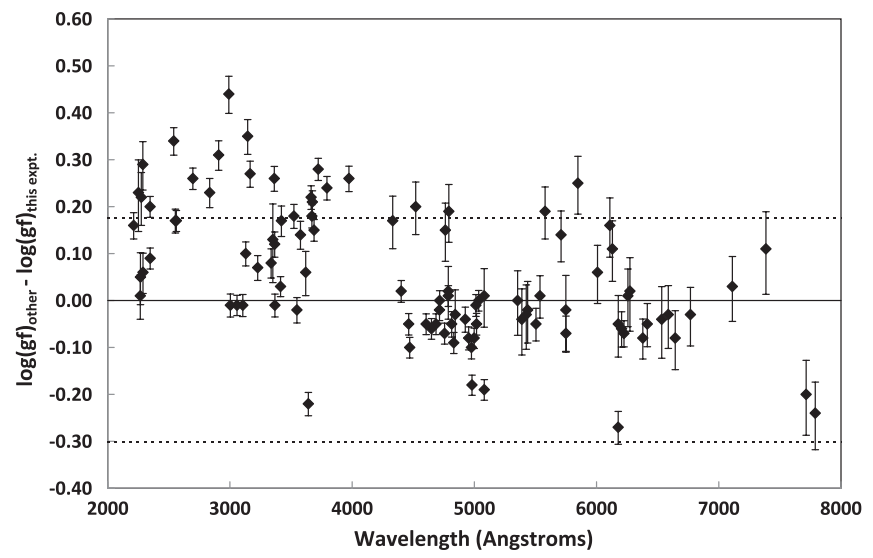

Figure 7. Comparison of $\log (g f)$ s with accuracy rank $\mathrm{D}(\leqslant 50 \%)$ and $\mathrm{D}+(\leqslant 40 \%)$ from the online NIST Atomic Spectra Database by Kramida et al. (2013) (also in text by Fuhr et al. 1988) to results of this study as a function of wavelength for 100 lines in common. The solid horizontal line indicates perfect agreement and the dashed lines indicate $\pm 50 \%$ differences. The error bars are from this work only.

with accuracy grades of $\mathrm{C}+(\leqslant 18 \%)$ and $\mathrm{C}(\leqslant 25 \%)$. Individual error bars represent uncertainties from this work, and the dotted lines represent $\pm 25 \%$ differences in $f$-values. The central line represents perfect agreement at a logarithmic difference of 0 . 


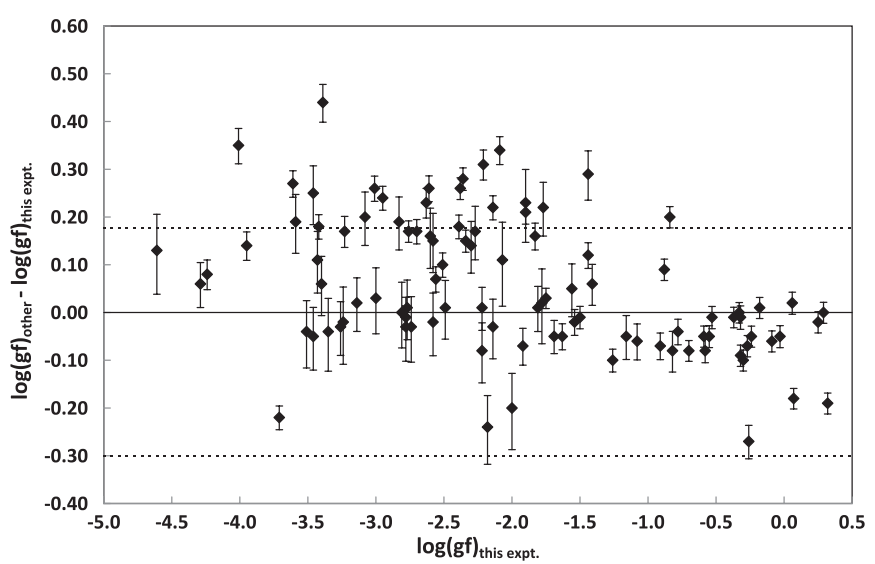

Figure 8. Comparison of $\log (g f)$ s for the same 100 lines shown in Figure 7, this time plotted as a function of $\log (g f)$ from this study. See Figure 7 for additional details.

There is generally good agreement, with $>80 \%$ of lines agreeing within combined uncertainties. Figure 6 shows the same 131 lines plotted as a function of the $\log (g f)$ measured in this work, with the lines and error bars having the same meaning as in Figure 5.

Figure 7 compares 100 lines in common between this work and the 2013 NIST Atomic Spectra Database with accuracy grades of D+ $(\leqslant 40 \%)$ and $\mathrm{D}(\leqslant 50 \%)$ as a function of wavelength. The central line and the error bars have the same meanings as in Figure 5. The dotted lines in Figure 7 represent $\pm 50 \%$ differences in $f$-values. Again there is good agreement between this work and the database, with $84 \%$ of lines agreeing within combined uncertainties. Figure 8 shows the same 100 lines plotted in Figure 7 as a function of the $\log (g f)$ measured in this work, with the lines and error bars having the same meaning as in Figure 7.

\section{THE NICKEL ABUNDANCE IN THE SOLAR PHOTOSPHERE}

We apply our new Ni I transition probability data to redetermine the $\mathrm{Ni}$ abundance of the solar photosphere. Our analytical procedure is nearly identical to that used in Lawler et al. (2013) and Wood et al. (2013). The main difference with our past papers is that we include isotopic line shifts for Ni I lines when such data are available (see the Appendix) and they appreciably affect total line broadening.

As in previous papers by our group, we first compute relative absorption strengths of Ni I lines as

$$
\mathrm{STR}=\log (g f)-\theta \chi
$$

with the $\log (g f)$ given in Table 3 , excitation energies $\chi(\mathrm{eV})$, and inverse temperature $\theta=5040 / \mathrm{T}$ (we assume $\theta=1.0$ for this rough calculation). The STR values are plotted as a function of wavelength in Figure 9. Red circles call attention to those lines that we use in the solar abundance computations. These relative strengths apply only to Ni I transitions and cannot be compared to those of Ni II or to species of other elements because they do not include elemental abundances and Saha ionization factors. However, to a good approximation, the line strengths for a single species depend on the transition probabilities and the Boltzmann excitation factors that make up the STR factors. In Figure 9 we place a horizontal line at $\mathrm{STR}=-5.75$. Examination of several

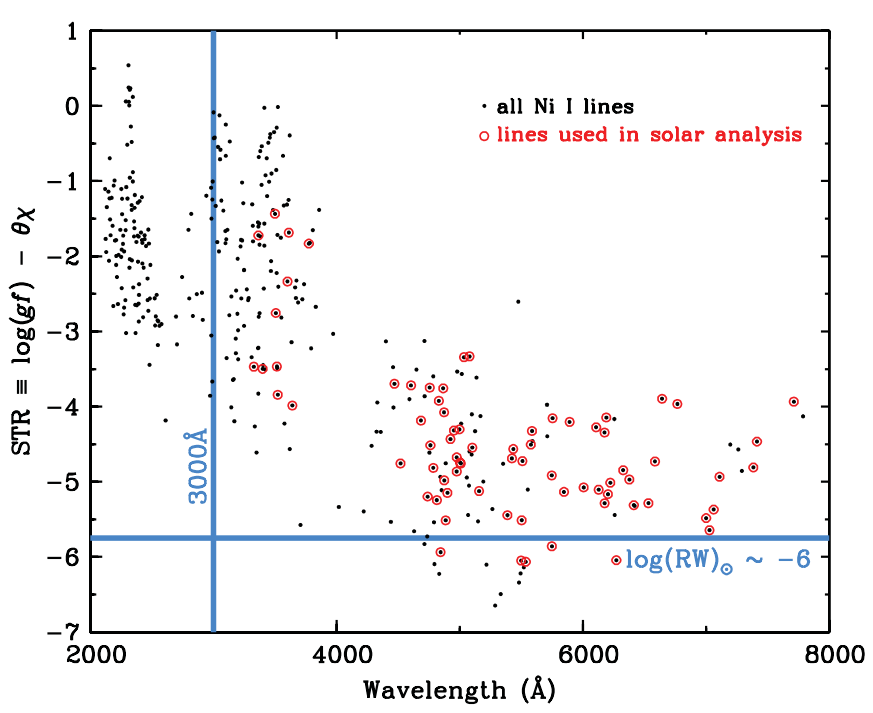

Figure 9. Relative strengths of Ni I lines of this study, plotted as a function of their wavelengths; see text for definitions of terms. The vertical blue line denotes the atmospheric cutoff wavelength. The horizontal blue line denotes the approximate strength of barely detectable Ni I lines (reduced widths $\log (\mathrm{RW})=-6)$. Red circles indicate lines employed in our solar photospheric abundance analysis.

(A color version of this figure is available in the online journal.)

solar photospheric Ni I lines (see below) suggests that lines of this strength will have reduced widths $\log (\mathrm{RW})=\log (\mathrm{EW} / \lambda) \sim$ -6 , approaching the weak-line limit of features that are useful in a solar abundance analysis. Only 14 of our Ni I lines have STR $<-5.75$. Like similar STR plots in our previous papers, the strongest lines are at shorter wavelengths; for $\mathrm{Ni}$ I they occur exclusively below $4000 \AA$.

Since almost all of the Ni I transitions in this study should be stronger than the weak-line limit, we consider the entire set of 371 lines for the determination of a new solar $\mathrm{Ni}$ abundance. We first visually inspect each line in the electronic version ${ }^{9}$ of the solar center-of-disk spectrum (Delbouille et al. 1973), and consult the Moore et al. (1966) solar line identification compendium. This procedure results in elimination of a few transitions whose photospheric line strengths are undetectably weak, and many more that are significantly compromised (especially for $\lambda<4000 \AA$ ) by blending with various atomic and/or molecular species. We are left with nearly $100 \mathrm{Ni}$ I lines deserving of more careful study.

We compute synthetic spectra for these surviving transitions and compare them to the Delbouille et al. (1973) solar spectrum. The synthetic spectra are convolved with Gaussian broadening functions to reproduce the combined effects of the very narrow spectrograph instrumental profile (resolving power, $R=\lambda / \Delta \lambda>$ $5 \times 10^{5}$ ) and solar macroturbulence. Spectrum line list construction is described by Lawler et al. (2013) in detail. Atomic and molecular lines in small spectral regions surrounding individual Ni I lines are assembled from the Kurucz (2011) database. ${ }^{10}$ The transition probabilities are updated with recent lab results, including the Ti I and Ti II data from Lawler et al. (2013) and Wood et al. (2013), and adjustments are made to $\log (g f)$ values of lines with no lab data in order to best reproduce the solar spectrum. We input these lists and the Holweger \& Müller (1974) empirical model photosphere to the current version of the LTE

\footnotetext{
9 http://bass2000.obspm.fr/solar_spect.php

$10 \mathrm{http} / / /$ kurucz.harvard.edu/linelists.html
} 


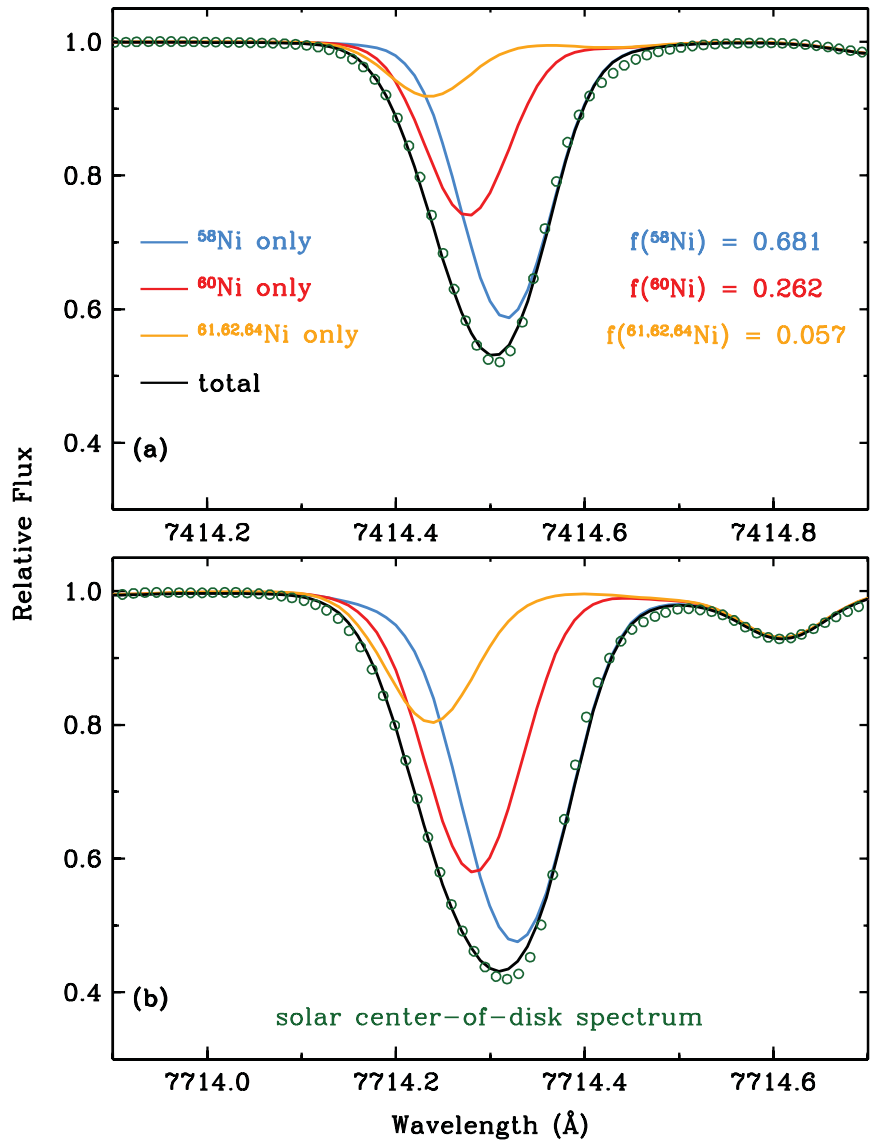

Figure 10. Synthetic and observed photospheric spectra of two Ni I lines that have significant isotopic substructure. The green open circles represent every sixth point from the Delbouille et al. (1973) solar center-of-disk spectrum. The lines representing syntheses of individual isotopic components and the combined synthesis are identified in the legend in panel (a). See the text for more information on the ${ }^{61,62,64} \mathrm{Ni}$ component.

(A color version of this figure is available in the online journal.)

line analysis code MOOG $^{11}$ (Sneden 1973). The comparison of observed and synthetic spectra result in the elimination of more lines due to unacceptably large blending of the $\mathrm{Ni}$ I lines by other transitions.

The final set of 76 lines are then subjected to a full synthetic spectrum analysis, this time with the inclusion of isotopic substructure in the computations when available from lab studies (see the Appendix). Isotopic fractions of the five stable Ni isotopes are (Coursey et al. 2010$)^{12}: f\left({ }^{58} \mathrm{Ni}\right)=0.6808, f\left({ }^{60} \mathrm{Ni}\right)=$ $0.2622, f\left({ }^{61} \mathrm{Ni}\right)=0.0114, f\left({ }^{62} \mathrm{Ni}\right)=0.0363$, and $f\left({ }^{64} \mathrm{Ni}\right)=$ 0.0093 . The three heaviest isotopes account for only $5.7 \%$ of the total solar-system $\mathrm{Ni}$ content. For ${ }^{58} \mathrm{Ni}$ and ${ }^{60} \mathrm{Ni}$ wavelengths we use the data given in the Appendix. However, there are far fewer lab data on shifts of the minor isotopes than on shifts of ${ }^{58} \mathrm{Ni}$ and ${ }^{60} \mathrm{Ni}$. Fortunately, Ni I isotopic line shifts depend almost linearly on nuclear mass. Therefore in our syntheses we approximate $61,62,64 \mathrm{Ni}$ as a single isotope and assign it an isotopic fraction of 0.057 . For a given line, we assume that the wavelength of this minor isotope is shifted from $\lambda\left({ }^{60} \mathrm{Ni}\right)$ by the same amount and in the same direction that $\lambda\left({ }^{60} \mathrm{Ni}\right)$ is shifted from $\lambda\left({ }^{58} \mathrm{Ni}\right)$.

In Figure 10 we show sample synthetic spectra for $\mathrm{Ni}$ I $7414.5 \AA$ and $7714.3 \AA$. These were chosen for display because

\footnotetext{
11 Available at http://www.as.utexas.edu/ chris/moog.html.

12 http://www.nist.gov/pml/data/comp.cfm
}

Table 4

Solar Photospheric Nickel Abundances from Individual Ni I Lines

\begin{tabular}{lcccc}
\hline \hline $\begin{array}{l}\text { Wavelength in Air } \\
(\AA)\end{array}$ & $\begin{array}{c}\text { Lower Energy } \\
(\mathrm{eV})\end{array}$ & $\log _{10}(g f)$ & $\log _{10}(\varepsilon)$ & Isotope $^{\mathrm{a}}$ \\
\hline 3328.713 & 0.109 & -3.36 & 6.25 & No \\
3365.765 & 0.422 & -1.30 & 6.28 & No \\
3401.164 & 3.417 & -0.08 & 6.15 & No \\
3500.851 & 0.165 & -1.27 & 6.23 & No \\
3507.693 & 0.165 & -2.59 & 6.25 & No \\
\hline
\end{tabular}

Notes.

a This column indicates whether isotopic substructure has been included in the synthetic spectrum of a line.

(This table is available in its entirety in a machine-readable form in the online journal. A portion is shown here for guidance regarding its form and content.)

they have some of the largest isotopic splitting of any of the lines considered in the present work. The larger widths of the total line profiles compared to those of the individual isotopic components can be easily seen in the figure. The main effect of the inclusion of isotopic subcomponents is to desaturate the transition. This leads to smaller derived abundances for strong Ni I lines with respect to those that are derived with single-line approximations. The magnitude of the decrease varies with isotopic splitting and line strength, but ranges from $0.01-0.08$ dex for the lines of this study. Note that isotopic shifts are much smaller for lines in the blue-UV spectral domain. We ignored isotopic effects for lines with $\lambda(58 \mathrm{Ni})-\lambda(60 \mathrm{Ni})<0.01 \AA$; the abundance changes for such lines are too small to be detected in our analysis.

The abundances from individual lines are listed in Table 4, in which we also record line wavelengths, excitation energies, oscillator strengths, and notes on whether isotopic substructure is included in the synthetic spectra computations. The line abundances are plotted as functions of wavelengths, excitation energies, and transition probabilities in Figure 11. There are no obvious trends beyond a small offset for the lines in the E.P. $=1.5-2.0 \mathrm{eV}$ range compared with other Ni I lines. There are 33 of the 76 transitions in the E.P. $=1.5-2.0 \mathrm{eV}$ range and all have wavelengths in the visible or near-IR spectral region, and are all quite weak, with branching fractions between 0.003 and 0.0001 (mean $\sim 0.001$ ). The less crowded spectral region of these lines and their lack of saturation makes them very convenient in abundance measurements. Unfortunately, their $\log (g f)$ measurements are exceptionally difficult. The dominant branches from the upper levels of these 33 transitions are in the UV. This requires a relative radiometric calibration over a large wavenumber range and, as always, uncertainty migrates to the weak branches. Furthermore, it is necessary to bootstrap line ratio measurements through a sequence of line pairs in spectra recorded with lower and lower currents in order to measure branching fractions for these weak lines with good $\mathrm{S} / \mathrm{N}$ and without optical depth errors. The resulting transition probabilities of these 33 lines have uncertainties of $\sim 15 \%$ ( $\sim 0.065$ dex $)$. This is near the "edge" of our lab measurement capabilities.

Abundance values from the new Ni I data suggest a possible small non-LTE effect in the solar photosphere for Ni I lines with E.P. $=1.5-2.0 \mathrm{eV}$. However, this effect is only one to two times larger than the uncertainties on the transition probabilities, and there are some additional uncertainties from the abundance determinations. Thus our data may hint at, but certainly do not demonstrate, a small non-LTE effect in solar photospheric Ni I line formation. To our knowledge, non-LTE 

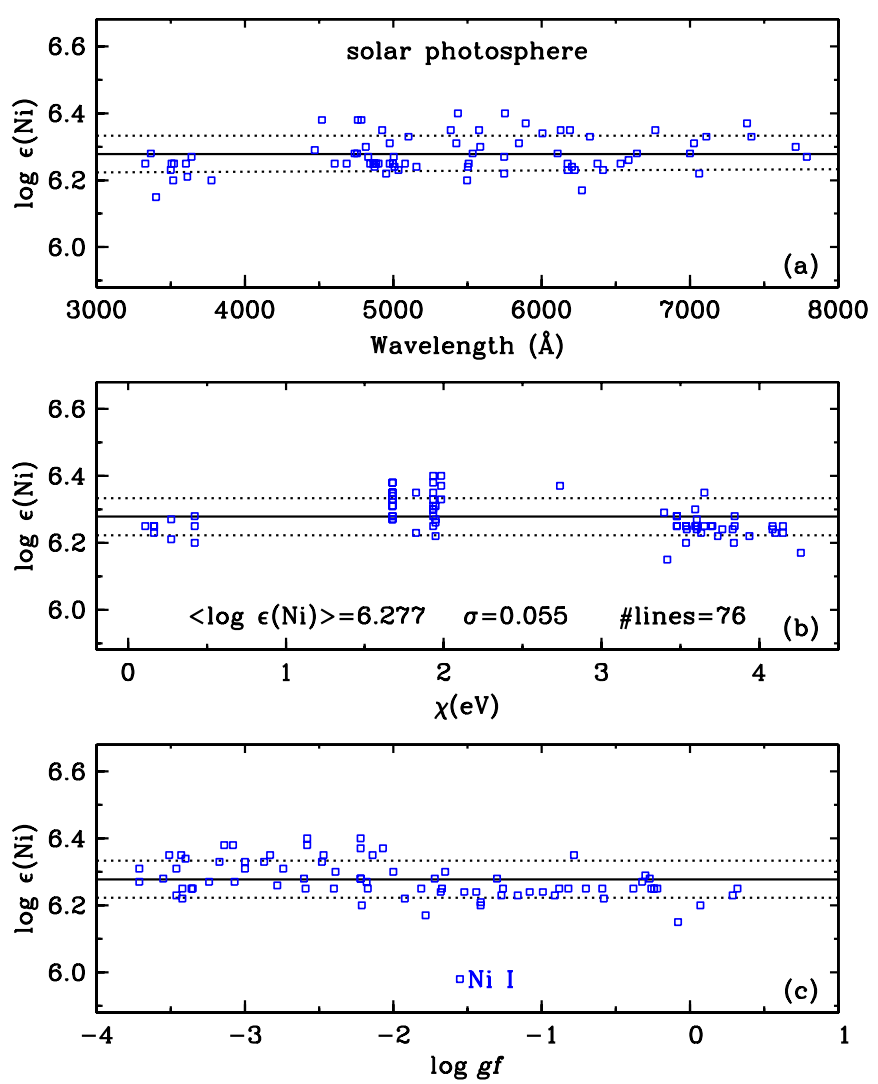

Figure 11. Solar photospheric abundances of Ni I lines plotted as functions of wavelength (a), lower excitation energy (b), and oscillator strength (c). The solid horizontal line represents the mean abundance, and the two dotted lines are placed $\pm 1 \sigma$ from the mean.

(A color version of this figure is available in the online journal.)

modeling has not been extensively explored. A few studies have specifically considered the $6769.64 \AA$ intercombination line, which is important for helioseismology (e.g., Bruls 1993). Recently, Vieytes \& Fontenla (2013) have provided an improved $\mathrm{Ni}$ I atomic model that can aid non-LTE investigations. Such studies should be pursued in the future.

From the total set of 76 lines we derive a new solar photospheric Ni abundance: $\langle\log \varepsilon(\mathrm{Ni})\rangle=6.277 \pm 0.004$ with $\sigma=0.055$. The mean abundance from Ni I is only slightly larger than the recommended solar photospheric abundances of $6.22 \pm 0.04$ (Asplund et al. 2009) and 6.23 \pm 0.04 (Lodders et al. 2009). As in our past studies, our mean abundance incorporates all 76 lines without any weighting.

\section{THE NICKEL ABUNDANCE OF METAL-POOR STAR HD 84937}

Fe-group $(21 \leqslant Z \leqslant 30)$ abundances in low-metallicity stars hold vital clues to early Galactic nucleosynthesis from massive stars. Theory can often predict detailed abundance distributions of these elements (e.g., Kobayashi et al. 2006). However, most abundance studies of these elements in actual metal-poor stars may not be reliable because: (1) the neutral species of Fe-group elements are most easily detected in visible $(\lambda>4000 \AA)$ spectra, yet the vast majority of these elements exist in the singly ionized state; and (2) there are concerns about the reliability of standard analytical assumptions (e.g., LTE, plane-parallel geometry) used in deriving abundances. These concerns need to be attacked on many fronts. The work of our group, first on rare-earth
Table 5

Nickel Abundances from Individual Ni I Lines in HD 84937

\begin{tabular}{lccc}
\hline \hline $\begin{array}{l}\text { Wavelength in Air } \\
(\AA)\end{array}$ & $\begin{array}{c}\text { Lower Energy } \\
(\mathrm{eV})\end{array}$ & $\log _{10}(g f)$ & $\log _{10}(\varepsilon)$ \\
\hline 2318.777 & 0.422 & -1.73 & 3.87 \\
2329.971 & 0.275 & +0.00 & 3.75 \\
2338.496 & 0.025 & -2.03 & 4.00 \\
2376.018 & 0.109 & -1.70 & 3.93 \\
2401.842 & 0.165 & -1.10 & 3.90 \\
\hline
\end{tabular}

(This table is available in its entirety in a machine-readable form in the online journal. A portion is shown here for guidance regarding its form and content.)

elements and now the Fe-group, is concentrating on increasing the quality and quantity of basic transition probability data.

The strongest lines of all Fe-group species lie in the UV spectral region, but this wavelength domain is very line-rich even in metal-poor giant stars. Therefore, as in our studies of Ti I and Ti II transition probabilities (Lawler et al. 2013; Wood et al. 2013), we concentrate on exploring the spectrum of HD 84937, a metal-poor main-sequence turnoff star $\left(T_{\text {eff }}=6300 \mathrm{~K}, \log \right.$ $g=4.0,[\mathrm{Fe} / \mathrm{H}]=-2.15$, and $\left.v_{\mathrm{t}}=1.5 \mathrm{~km} \mathrm{~s}^{-1}\right)$. While lines in the visible spectral region of HD 84937 are usually very weak, they are stronger and more numerous in the UV. Additionally, the number of ionized-species transitions is greatly increased, allowing tests of the Saha ionization balance in several elements.

We derive the Ni abundance in HD 84937 in the same manner as in our previous Ti I and Ti II studies. See Lawler et al. (2013) for a detailed description of the optical ESO VLT UVES and HST/STIS UV high-resolution spectra. These combined spectra yield continuous coverage from $2300 \AA$ to $6800 \AA$. We select viable Ni I transitions in the HD 84937 spectrum and derive line-by-line abundances as done in Lawler et al. (2013). In these computations we neglect isotopic splitting for two reasons. First, all lines of Ni I in the visible wavelength region $(\lambda>4000 \AA)$ are weak and thus abundances derived from them are unaffected by isotopic broadening of the line profiles. Second, although the lines are often stronger at shorter wavelengths, the isotopic wavelength splits are very small. The mean difference in the UV is $\langle|\lambda(58 \mathrm{Ni})-\lambda(60 \mathrm{Ni})|\rangle=0.002 \AA$, undetectable with our data.

Line-by-line abundance information for the $77 \mathrm{Ni}$ lines analyzed in HD 84937 is given in Table 5, and in Figure 13 we plot the abundances as functions of their wavelengths, excitation energies, and transition probabilities. There are no obvious abundance trends with any of these quantities. In particular, the Ni I data do not show a systematic decrease in abundance in the Balmer continuum region (approximately $3100-3650 \AA$ ) that is seen in HD 84937 for Fe I by Lawler et al. (2013) and for Ti II by Wood et al. (2013). We derive a mean abundance $\langle\log \varepsilon(\mathrm{Ni})\rangle=3.888 \pm 0.008$ with $\sigma=0.068$.

$\mathrm{A}$ few $\mathrm{Fe}$-group elements (e.g., $\mathrm{Ti}$ and $\mathrm{Fe}$ ) are represented by many detectable transitions of both neutral and ionized species that are accessible to ground-based high-resolution spectroscopy. Unfortunately, most Ni II lines with extant laboratory transition probability measurements are in the vacuum-UV wavelength region. Moore et al. (1966) list 23 lines of Ni II in the range $2988-4362 \AA$, but all of them arise from high excitation energy levels $(\chi \geqslant 2.9 \mathrm{eV})$, almost all are weak and/or blended in the solar spectrum, and none have reliable $\log (g f)$ values. Therefore we ignore Ni II in our solar analysis. However, the HST/STIS spectrum of HD 84937 exhibits many lines of this species that were subjected to a lab analysis by Fedchak \& Lawler (1999). There are 19 transitions from their study in the 


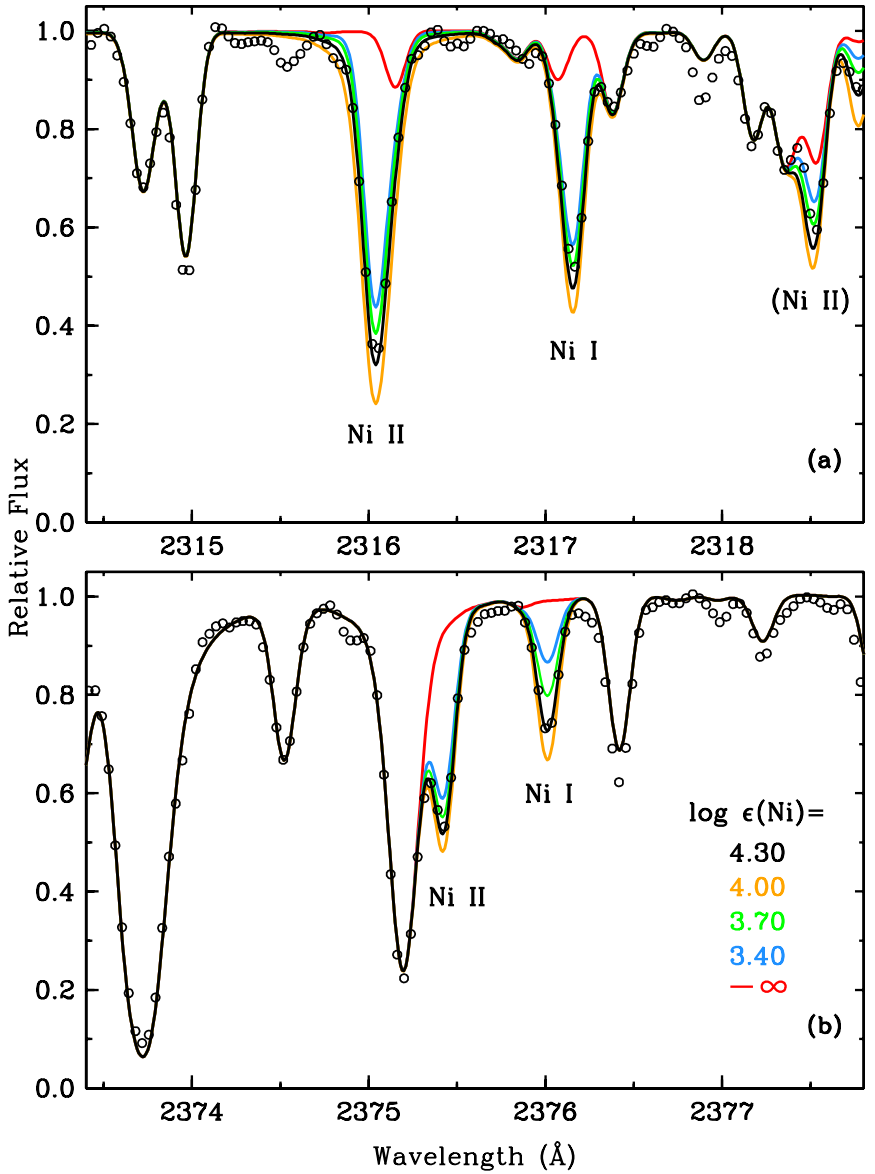

Figure 12. Observed and synthetic spectra of two small wavelength intervals in the UV spectrum of HD 84937 that contain both Ni I and Ni II lines used in this study. The observed HST/STIS spectrum is shown with open circles. The synthetic spectra have been computed for abundances that are color-coded as given in the legend of panel (b). The synthesis labeled " $-\infty$ " is computed without any $\mathrm{Ni}$ contribution. The line labeled "(Ni II)" in panel (a) was not included in the lab study of Fedchak \& Lawler (1999).

(A color version of this figure is available in the online journal.)

$H S T /$ STIS spectral range $(\lambda>2300 \AA)$. In Figure 12 we display observed and synthetic spectra of two such lines. These are chosen to also contain lines of Ni I from the present study. It is important to note that lines of $\mathrm{Ni}$ II are all relatively strong, and thus derived abundances from them are sensitive to our adopted microturbulence value for HD 84937. However, the Ni I lines in the $H S T$ /STIS spectra are also typically strong, and so changing the microturbulence will affect both species in the same fashion, leaving abundance ratios derived from $\mathrm{Ni}$ I and $\mathrm{Ni}$ II lines relatively unchanged.

We can identify eight usable Ni II transitions in HD 84937, whose parameters and abundance estimates are given in Table 6, and we show them (along with the Ni I line results) in Figure 13. For these lines we derive $\langle\log \varepsilon(\mathrm{Ni})\rangle=3.89 \pm 0.04$ with $\sigma=$ 0.10 , in excellent agreement with the mean abundance from $\mathrm{Ni}$ I. Given the small number of $\mathrm{Ni}$ II transitions used, we cannot draw firm conclusions from the concordance between the $\mathrm{Ni}$ species abundances, but with the present data we do not see evidence of major departures from LTE in the ionization balance for Ni in HD 84937. Further lab studies of weaker Ni II transitions in the visible spectral region would be welcome.

Following the discussion of Lawler et al. (2013), we estimate that internal line-to-line scatter uncertainties are $\leqslant 0.04 \mathrm{dex}$. For external error estimates, we derive abundances of typical $\mathrm{Ni}$ I
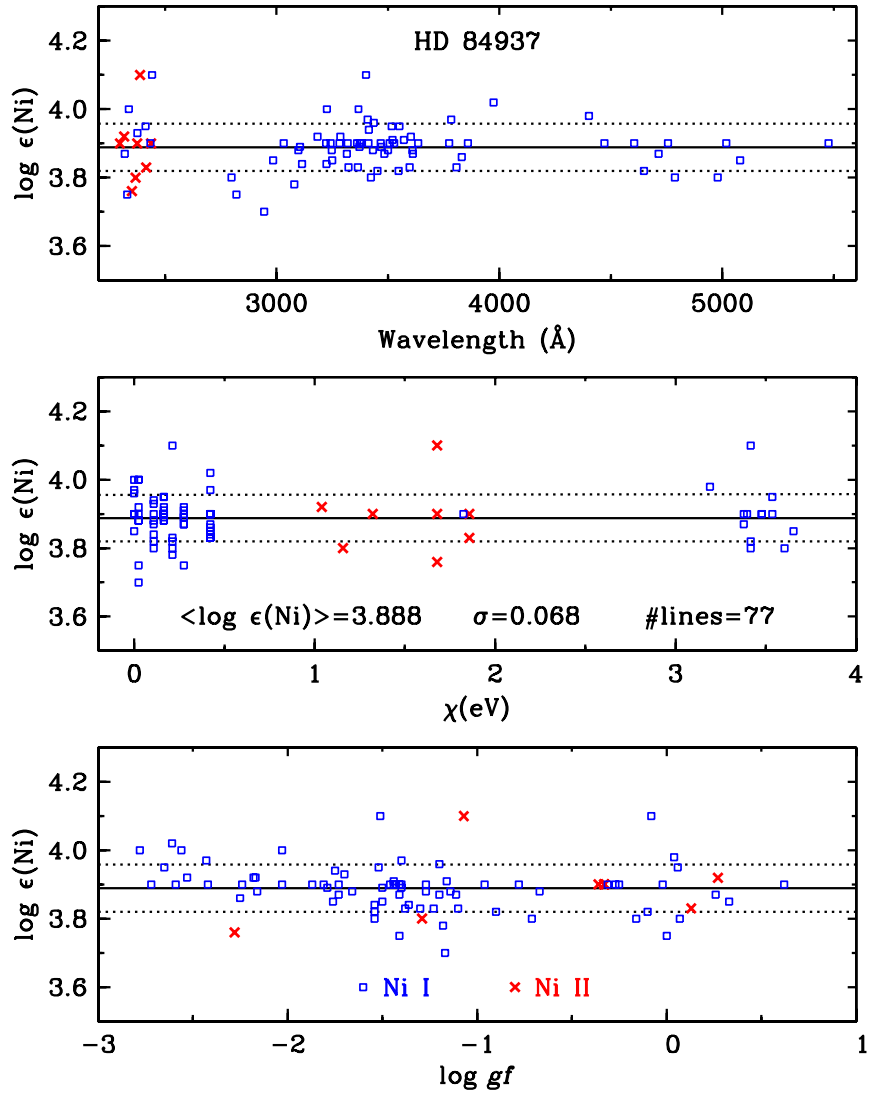

Figure 13. Abundances derived from Ni I and Ni II lines in HD 84937, plotted as functions of wavelength (a), lower excitation energy (b), and oscillator strength (c). The solid horizontal line represents the mean abundance, and the two dotted lines are placed $\pm 1 \sigma$ from the mean.

(A color version of this figure is available in the online journal.)

Table 6.

Nickel Abundances from Individual Ni II Lines in HD 84937

\begin{tabular}{lcrc}
\hline \hline $\begin{array}{l}\text { Wavelength in Air } \\
(\AA)\end{array}$ & $\begin{array}{c}\text { Lower Level } \\
(\mathrm{eV})\end{array}$ & $\log _{10}(g f)$ & $\log _{10}(\varepsilon)$ \\
\hline 2297.49 & 1.321 & -0.33 & 3.90 \\
2316.04 & 1.040 & 0.27 & 3.92 \\
2350.85 & 1.679 & -2.28 & 3.76 \\
2367.38 & 1.156 & -1.29 & 3.80 \\
2375.42 & 1.858 & -0.36 & 3.90 \\
2387.76 & 1.679 & -1.07 & 4.10 \\
2416.14 & 1.858 & 0.13 & 3.83 \\
2437.89 & 1.679 & -0.33 & 3.90 \\
\hline
\end{tabular}

and $\mathrm{Ni}$ II lines with model atmosphere parameters varied in accord with the HD 84937 uncertainties. For Ni I we obtain: $\Delta(\log \varepsilon) \approx+0.12$ for $\Delta\left(T_{\text {eff }}\right)=+150 \mathrm{~K} ; \Delta(\log \varepsilon) \approx-0.02$ for $\Delta(\log g)=+0.3 ; \Delta(\log \varepsilon) \approx-0.01$ for $\Delta([\mathrm{M} / \mathrm{H}])=-0.3$; and $\Delta(\log \varepsilon) \approx+0.00$ to +0.15 for $\Delta\left(v_{\mathrm{t}}\right)=-0.25$, depending on whether the lines are on the linear or saturated part of the curveof-growth. The responses of $\mathrm{Ni}$ I line abundances to temperature and gravity changes are understandable from Saha ionization balances of both $\mathrm{Ni}$ I and $\mathrm{H}^{-}$, and they are essentially identical to those of $\mathrm{Fe}$ I because the $\mathrm{Ni}$ and $\mathrm{Fe}$ ionization energies differ by only $0.3 \mathrm{eV}$. Thus, to the extent that LTE conditions apply to the line formation of these two elements, $[\mathrm{Ni} / \mathrm{Fe}]$ has little dependence on the assumed $\mathrm{T}_{\text {eff }}$ and $\log (g)$ parameters for HD 84937. Note also that the assumed microturbulent velocity does not influence the Ni abundance much since most Ni I lines in the visible spectral region are very weak. 
We repeat the model parameter variation for the UV spectral region where Ni II lines are detected. There is no substantial difference from the visible to the UV in the responses of $\mathrm{Ni}$ I lines to model atmospheric parameter changes. For Ni II: $\Delta(\log \varepsilon) \approx+0.06$ for $\Delta\left(T_{\text {eff }}\right)=+150 \mathrm{~K} ; \Delta(\log \varepsilon) \approx-0.09$ for $\Delta(\log g)=+0.3 ; \Delta(\log \varepsilon) \approx-0.01$ for $\Delta([\mathrm{M} / \mathrm{H}])=-0.3$; and $\Delta(\log \varepsilon) \approx+0.00$ to +0.25 for $\Delta\left(v_{\mathrm{t}}\right)=-0.25$, depending on whether the lines are on the linear or saturated part of the curve-of-growth. However, as mentioned above, both Ni I and Ni II lines in the UV have comparable strengths, muting the dependence on assumed microturbulent velocity when comparing the abundances from these two Ni species.

Here we have shown that the Ni abundance in HD 84937 is well-determined from $\mathrm{Ni}$ I and $\mathrm{Ni}$ II lines ranging from the visible to the UV spectral region. We cannot detect departures from LTE in the Ni ionization equilibrium. However, we repeat the cautions of Wood et al. (2013) and others: we have not investigated the statistical equilibrium of $\mathrm{Ni}$ without the restrictive assumptions of LTE and one-dimensional atmospheric models. We cannot quantify their influence on derived $\mathrm{Ni}$ abundances for this star, and urge future detailed consideration of such issues.

\section{IMPLICATIONS FOR Fe-GROUP NUCLEOSYNTHESIS}

Our group has been working to determine precise atomic parameters of Fe-group species in order to confront more directly the nucleosynthetic origin of these elements. Recent results include: for Mn I and Mn II, Den Hartog et al. (2011); for Ti I, Lawler et al. (2013); for Ti II, Wood et al. (2013); and $\mathrm{Ni}$ I (this paper, which also includes a new look at Ni II). We are using the new experimental atomic data to derive accurate abundances of these elements in metal-poor halo stars, similar to our efforts for the rare-earth elements (REE; see, e.g., Den Hartog et al. 2006; Lawler et al. 2007, 2008, 2009; Sneden et al. 2009). Such well-determined experimental data for the Fe-group elements have been lacking in the past, leading to significant stellar abundance uncertainties.

The Fe-group elements are formed in core-collapse $\mathrm{SNe}$ explosions (see Thielemann et al. 1996) and then ejected into space where they are incorporated into new stars, including into halo stars early in the history of the Galaxy. Thus, observations of these elements in halo stars provide insight into early Galactic star formation and element nucleosynthesis. In addition, the Fegroup elements are synthesized in a different manner (chargedparticle synthesis) than the REE (neutron-capture processes), and thus the observational abundances of these two different element groups sample different regimes of the halo progenitors. Specifically, the Fe-group elements are formed in the siliconburning region of the exploding SNe. Some of these elements, such as $\mathrm{Ni}$, are (mostly) synthesized as a result of complete silicon fusion. In other cases, such as for $\mathrm{Ti}$, the temperature in the region never rises high enough, instead leading to incomplete Si fusion. The details of how this nucleosynthesis occurs depend critically upon the SN parameters, including the location of the mass cut (the point in the star where the mass is ejected), the explosion energy, the mass of the SN progenitor, and the neutron excess (e.g., Nakamura et al. 1999). Thus, precise stellar abundance values can constrain the conditions that occur in the nucleosynthesis zones of SN, and thereby provide insights into how $\mathrm{SNe}$ explode.

It should also be noted that while $\mathrm{SNe}$ are responsible for Fe-group element synthesis, there is no clear consensus on the astrophysical site for rapid neutron-capture synthesis (the $r$-process), which is the regime where the REE are produced.

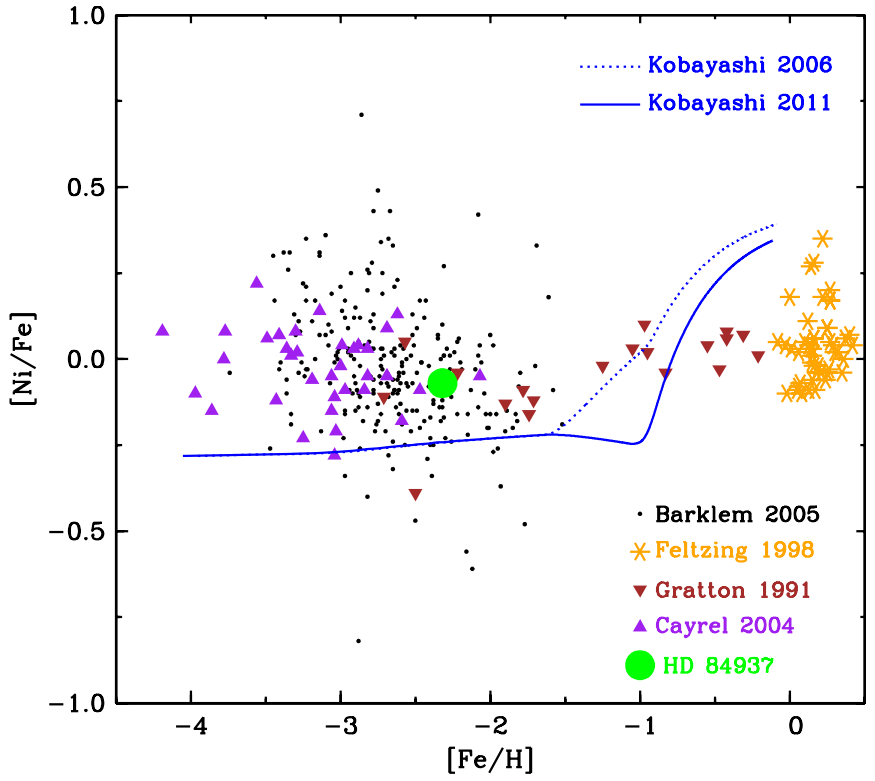

Figure 14. $[\mathrm{Ni} / \mathrm{Fe}]$ abundance ratios as a function of $[\mathrm{Fe} / \mathrm{H}]$ metallicities. Observed abundances are included from four literature surveys as identified in the figure legend and the text, and our new value for HD 84937 is shown as a large green dot. The theoretical abundance predictions (blue lines) are from Kobayashi et al. $(2006,2011)$ as identified in the figure legend.

(A color version of this figure is available in the online journal.)

Previous work has suggested neutron-star binary mergers and neutron-star winds (rather than $\mathrm{SNe}$ ) as the site for this neutroncapture synthesis (Lattimer \& Schramm 1974, 1976; Rosswog et al. 1999; Janka et al. 1999; Wanajo \& Janka 2012). Thus comparisons among the stellar observations of the REE and the $\mathrm{Fe}$-group elements might in fact be sampling nucleosynthesis results from two different sites. Such observational constraints and comparisons might provide further clues about the sites for the earliest heavy element synthesis.

Using the $\mathrm{Ni}$ abundance we find for HD 84937 of $\log \varepsilon(\mathrm{Ni})=$ 3.89 with our new solar value of $\log \varepsilon(\mathrm{Ni})=6.28$ yields $[\mathrm{Ni} / \mathrm{H}]=-2.39$ for this star. The Fe abundance of HD 84397 has recently been re-determined, yielding $\log \varepsilon(\mathrm{Fe})=5.18$ and a metallicity of $[\mathrm{Fe} / \mathrm{H}]=-2.32$ (Lawler et al. 2013). In that paper, the authors derive a relative titanium abundance of $[\mathrm{Ti} / \mathrm{Fe}]=$ +0.47 . Our new results yield values of $[\mathrm{Ni} / \mathrm{Fe}]=-0.07$ and $[\mathrm{Ti} / \mathrm{Ni}]=+0.54$ for HD 84937. Lawler et al. (2013) argue that the majority of SNe models have difficulty in reproducing the observed Ti abundance in HD 84937, as seen in other metal-poor stars (e.g., Kobayashi et al. 2006). Our new precise measurement of [Ti/Ni] will provide further constraints on SN models. ${ }^{13}$

Our new Ni abundance result for HD 84937 provides one new data point that can be placed in the context of Galactic chemical evolution. Models that predict mean abundance ratios as a function of metallicity depend sensitively on a number of parameters including the initial mass function and $\mathrm{SN}$ yields. Our value of $[\mathrm{Ni} / \mathrm{Fe}]=-0.07$ can be compared to previous determinations of $[\mathrm{Ni} / \mathrm{Fe}]$ as a function of $[\mathrm{Fe} / \mathrm{H}]($ see Figures 1 and 2 of Henry et al. (2010) for a compilation of various values from the literature). We show a similar observation/theory comparison in Figure 14, where we have plotted the $[\mathrm{Ni} / \mathrm{Fe}]$ ratios as a function of metallicity from

\footnotetext{
13 Complicating the theoretical interpretation is that while ${ }^{56} \mathrm{Ni}$ (which decays to ${ }^{56} \mathrm{Fe}$ ) is the dominant element for complete Si fusion, this element can also be synthesized in incomplete Si fusion (see Nakamura et al. 1999).
} 
Table 7

Shifted Isotopic Wavelengths for 303 Lines of Ni I Organized by Increasing Center of Gravity Wavelength in Air

\begin{tabular}{|c|c|c|c|c|c|c|c|c|}
\hline \multirow{2}{*}{$\begin{array}{l}\text { Wavelength }^{\mathrm{a}} \\
\text { in Air } \\
(\AA)\end{array}$} & \multicolumn{3}{|c|}{ Upper Level } & \multicolumn{3}{|c|}{ Lower Level } & \multirow{2}{*}{$\begin{array}{c}\text { Shifted }{ }^{58} \mathrm{Ni} \\
\text { Wavelength in Air } \\
(\AA)\end{array}$} & \multirow{2}{*}{$\begin{array}{c}\text { Shifted }{ }^{60} \mathrm{Ni} \\
\text { Wavelength in Air } \\
(\AA ̊)\end{array}$} \\
\hline & Energy $^{b}\left(\mathrm{~cm}^{-1}\right)$ & Parity & $J$ & Energy $^{\mathrm{b}}\left(\mathrm{cm}^{-1}\right)$ & Parity & $J$ & & \\
\hline 2121.3903 & 47328.784 & od & 2 & 204.787 & ev & 3 & 2121.3898 & 2121.3910 \\
\hline 2125.6261 & 47030.102 & od & 3 & 0.000 & ev & 4 & 2125.6265 & 2125.6254 \\
\hline 2129.9541 & 47139.337 & od & 2 & 204.787 & ev & 3 & 2129.9535 & 2129.9550 \\
\hline 2134.9235 & 47030.102 & od & 3 & 204.787 & ev & 3 & 2134.9228 & 2134.9244 \\
\hline 2147.7838 & 47424.785 & od & 1 & 879.816 & ev & 2 & 2147.7834 & 2147.7845 \\
\hline
\end{tabular}

Notes.

a Wavelength values computed from energy levels using the standard index of air from Peck \& Reeder (1972).

b Energy levels from the online 2013 NIST Atomic Spectra Database by Kramida et al.

(This table is available in its entirety in a machine-readable form in the online journal. A portion is shown here for guidance regarding its form and content.)

a number of sources including Gratton \& Sneden (1991), Feltzing \& Gustafsson (1998), Cayrel et al. (2004), and Barklem et al. (2005). Our new [Ni/Fe] value for HD 84937 is shown as a large green dot in Figure 14. There is a great deal of scatter at metallicities $[\mathrm{Fe} / \mathrm{H}]<-2$, some of which may be intrinsic, but some is probably the result of imprecise abundance values resulting from older and non-experimental atomic data. Superimposed on the plot are theoretical chemical evolution abundance curves from the work of Kobayashi et al. (2006) and Kobayashi et al. (2011). The plateau for these curves is based upon the chosen initial mass function and the yields from both $\mathrm{SNe}$ and hypernovae (HNe). The agreement with our new $[\mathrm{Ni} / \mathrm{Fe}]$ value for HD 84937 is very encouraging, differing by only +0.14 from these models. Nevertheless, even this small offset might serve as a further constraint on such models, suggesting slightly higher values of $[\mathrm{Ni} / \mathrm{Fe}]$ resulting from the yields of core-collapse $\mathrm{SNe}$ and $\mathrm{HNe}$ at low metallicities. At higher metallicities there is a significant increase in $[\mathrm{Ni} / \mathrm{Fe}]$ as a result of the increasing $\mathrm{Ni}$ and Fe production from Type Ia SNe. Clearly, more such precise abundance values will be required to understand early Galactic chemical evolution and constrain the various parameters (e.g., yields) and models.

\section{SUMMARY}

Accurate absolute transition probabilities are reported for 371 lines of Ni I from the UV through near-IR. Branching fractions are measured from a combination of archived FTS spectra and new data recorded with an echelle spectrograph. The use of the echelle spectrograph allows for optical depth errors in $\mathrm{Ni}$ I to be addressed and leads to an overall reduction in systematic uncertainties. These branching fractions are combined with previously reported radiative lifetime measurements to produce the absolute transition probabilities. Generally good agreement is found in comparison to previously reported $\mathrm{Ni}$ I transition probabilities. The new Ni I data are applied to re-determine the $\mathrm{Ni}$ abundance in the photospheres of the Sun and the metal-poor star HD 84937. There is good agreement with the mean of other $[\mathrm{Ni} / \mathrm{Fe}]$ observations in the metallicity regime of this star, and only a small discrepancy with theoretical predictions.

The authors acknowledge the contribution of N. Brewer on data analysis for this project. This work is supported in part by NASA grant NNX10AN93G (J.E.L.) and NSF grants AST0908978 and AST-1211585 (C.S.). This paper was completed while C.S. was on a University of Texas Faculty Research
Assignment, in residence at the Department of Astronomy and Space Sciences of Ege University. Financial support from the University of Texas and The Scientific and Technological Research Council of Turkey (TÜBITAK, project No. 112T929) are greatly appreciated. We thank Chiaki Kobayashi for providing us with her data, and thank her and Maria Bergemann for helpful comments.

\section{APPENDIX}

Isotopic wavelength shifts for 303 of the $371 \mathrm{Ni}$ I lines with measured transition probabilities in this study are compiled in Table 7. Previously reported measurements of the isotopic wavenumber splitting by Schroeder \& Mack (1961), Steudel et al. (1980), and Litzén et al. (1993) are combined and used to determine the shifted ${ }^{58} \mathrm{Ni}$ and ${ }^{60} \mathrm{Ni}$ energy levels using a least squares technique. All measured isotopic splits between the upper and lower Ni I levels appearing in Table 3 are included, with equal weights given to the three studies listed above. The shifted energy levels are used to calculate the shifted isotopic wavelengths in Table 7 using the standard index of air (Peck \& Reeder 1972). Due to a lack of available laboratory measurements, wavelength shifts for the minor isotopes ${ }^{61,62,64} \mathrm{Ni}$ are not included in Table 7. These isotopes together account for only $5.7 \%$ of the solar system $\mathrm{Ni}$ content. In cases where isotope shift data are used in abundance determinations, we approximate the ${ }^{61,62,64} \mathrm{Ni}$ isotopes as a single "isotope" and assign it an isotopic fraction of 0.057 . Since $\mathrm{Ni}$ isotopic wavelength shifts depend almost linearly on isotope mass, the wavelength for this combined "isotope" is shifted from $\lambda\left({ }^{60} \mathrm{Ni}\right)$ by the same amount and in the same direction that $\lambda\left({ }^{60} \mathrm{Ni}\right)$ is shifted with respect to $\lambda\left({ }^{58} \mathrm{Ni}\right)$. However, since this is only a rough approximation, the wavelengths for this combined "isotope" are not included in Table 7.

\section{REFERENCES}

Adams, D. L., \& Whaling, W. 1981, JOSA, 71, 1036

Asplund, M. 2005, ARA\&A, 43, 481

Asplund, M., Grevesse, N., Sauval, A. J., \& Scott, P. 2009, ARA\&A, 47, 481

Barklem, P. S., Christlieb, N., Beers, T. C., et al. 2005, A\&A, 439, 129

Bergeson, S. D., \& Lawler, J. E. 1993, JOSAB, 10, 794

Blackwell, D. E., Booth, A. J., \& Petford, A. D. 1989, MNRAS, 236, 235

Bonifacio, P., Spite, M., Cayrel, R., et al. 2009, A\&A, 501, 519

Brault, J. W. 1976, JOSA, 66, 1081

Bruls, J. H. M. J. 1993, A\&A, 269, 509

Cayrel, R., Depange, E., Spite, M., et al. 2004, A\&A, 416, 1117 
Coursey, J. S., Schwab, D. J., Tsai, J. J., \& Dragoset, R. A. 2010, Atomic Weights and Isotopic Compositions (version 3.0; Gaithersburg, MD: US NIST)

Cowan, J. J., Sneden, C., Spite, M., et al. 2002, ApJ, 572, 861

Danzmann, K., \& Kock, M. 1982, JOSA, 72, 1556

Delbouille, L., Roland, G., \& Neven, L. 1973, Photometric Atlas of the Solar Spectrum from $\lambda 3000$ to $\lambda 10000$ (Liège: Inst. d'Ap., Univ. de Liège)

Den Hartog, E. A., Lawler, J. E., Sneden, C., \& Cowan, J. J. 2006, ApJS, 167,292

Den Hartog, E. A., Lawler, J. E., Sobeck, J. S., Sneden, C., \& Cowan, J. J. 2011, ApJS, 194, 35

Fedchak, J. A., \& Lawler, J. E. 1999, ApJ, 523, 734

Feltzing, S., \& Gustafsson, B. 1998, A\&AS, 129, 237

Fuhr, J. R., Martin, G. A., \& Wiese, W. L. 1988, JPCRD, 17, Suppl. 4, 1

Gratton, R. G., \& Sneden, C. 1991, A\&A, 241, 501

Hashiguchi, S., \& Hasikuni, M. 1985, JPSJ, 54, 1290

Henry, R. B. C., Cowan, J. J., \& Sobeck, J. 2010, ApJ, 709, 715

Holweger, H., \& Müller, E. A. 1974, SoPh, 39, 19

Janka, H.-T., Eberl, T., Ruffert, M., \& Fryer, C. L. 1999, ApJ, 527, L39

Kobayashi, C., Karakas, A. I., \& Umeda, H. 2011, MNRAS, 414, 3231

Kobayashi, C., Umeda, H., Nomoto, K., Tominaga, N., \& Ohkubo, T. 2006, ApJ, 653, 1145

Kramida, A., Ralchenko, Yu., Reader, J., \& NIST ASD Team, 2013, NIST Atomic Spectra Database (version 5.1) (Gaithersburg, MD: US NIST)

Kurucz, R. L. 2011, CaJPh, 89, 417

Lai, D. K., Bolte, M., Johnson, J. A., et al. 2008, ApJ, 681, 1524

Lattimer, J. M., \& Schramm, D. N. 1974, ApJ, 192, L145

Lattimer, J. M., \& Schramm, D. N. 1976, ApJ, 210, 549

Lawler, J. E., Den Hartog, E. A., Labby, Z. E., et al. 2007, ApJS, 169, 120

Lawler, J. E., Guzman, A., Wood, M. P., Sneden, C., \& Cowan, J. J. 2013, ApJS, 205,11

Lawler, J. E., Sneden, C., Cowan, J. J., Ivans, I. I., \& Den Hartog, E. A 2009, ApJS, 182, 51

Lawler, J. E., Sneden, C., Cowan, J. J., et al. 2008, ApJS, 178, 71
Litzén, U., Brault, J. W., \& Thorne, A. P. 1993, PhyS, 47, 628

Lodders, K., Palme, H., \& Gail, H.-P. 2009, Landolt-Börnstein, 4B, 44L

McWilliam, A. 1997, ARA\&A, 35, 503

McWilliam, A., Preston, G. W., Sneden, C., \& Searle, L. 1995a, AJ, 109,2757

McWilliam, A., Preston, G. W., Sneden, C., \& Shectman, S. 1995b, AJ, 109, 2736

Moore, C. E., Minnaert, M. G. J., \& Houtgast, J. 1966, The Solar Spectrum $2935 \AA$ to $8770 \AA$ (NBS Monograph, Vol. 61; Washington, DC: US GPO)

Nakamura, T., Umeda, H., Nomoto, K., Thielemann, F.-K., \& Burrows, A. 1999, ApJ, 517, 193

Peck, E. R., \& Reeder, K. 1972, JOSA, 62, 958

Roederer, I. U. 2009, AJ, 137, 272

Rosswog, S., Liebendörfer, M., Thielemann, F.-K., et al. 1999, A\&A, 341, 499

Schroeder, D. J., \& Mack, J. E. 1961, PhRv, 121, 1726

Sneden, C. 1973, ApJ, 184, 839

Sneden, C., Cowan, J. J., Lawler, J. E., et al. 2003, ApJ, 591, 936

Sneden, C., Lawler, J. E., Cowan, J. J., Ivans, I. I., \& Den Hartog, E. A. 2009, ApJS, 182, 80

Steudel, A., Triebe, U., \& Wendlandt, D. 1980, ZPhyA, 296, 189

Suda, T., Yamada, S., Katsuna, Y., et al. 2011, MNRAS, 412, 843

Thielemann, F.-K., Nomoto, K., \& Hashimoto, M. A. 1996, ApJ, 460, 408

Thorne, A. P., Harris, C. J., Wynne-Jones, I., Learner, R. C. M., \& Cox, G. 1987, JPhE, 20, 54

Vieytes, M. C., \& Fontenla, J. M. 2013, ApJ, 769, 103

Wanajo, S., \& Janka, H.-T. 2012, ApJ, 746, 180

Westin, J., Sneden, C., Gustafsson, B., \& Cowan, J. J. 2000, ApJ, 530, 783

Whaling, W., Carle, M. T., \& Pitt, M. L. 1993, JQSRT, 50, 7

Wickliffe, M. E., \& Lawler, J. E. 1997, ApJS, 110, 163

Wickliffe, M. E., Lawler, J. E., \& Nave, G. 2000, JQSRT, 66, 363

Wood, M. P., \& Lawler, J. E. 2012, ApOpt, 51, 8407

Wood, M. P., Lawler, J. E., Sneden, C., \& Cowan, J. J. 2013, ApJS, 208, 27

Yong, D., Norris, J. E., Bessell, M. S., et al. 2013, ApJ, 762, 26 